\section{Three-dimensional steep wave impact on a vertical plate with an open}

\section{rectangular section}

\section{I.K. Chatjigeorgiou ${ }^{1}$}

School of Naval Architecture and Marine Engineering, National Technical University of Athens, Greece

School of Mathematics, University of East Anglia, Norwich, UK

\section{A.A. KorobKIN}

School of Mathematics, University of East Anglia, Norwich, UK

\section{M.J. COOKER}

School of Mathematics, University of East Anglia, Norwich, UK

\section{Abstract}

The present study treats the three-dimensional hydrodynamic slamming problem on a vertical plate subjected to the impact of a steep wave moving towards the plate with a constant velocity. The problem is complicated significantly by assuming that there is a rectangular opening on the plate which ils cons the disch ponditions which should be taken into account properly. The mathematical process assimilates the plate with a degerate elliptical cylinder allowing thus the employment of elliptical harmonics that plate we satisfaction of the free-surface boundary condition of the front face of the steep wave, beyond the plate. This assumption leads to an additional boundary value problem with mixed ceyond the plate. This assinption in the vertical direction. The associted problem in it is solved through a transformation into integral equations. To tackle the boundary value problem in it is solved through a transformation into tirection a perturbation technique is employed. Extensive numerical calculations are presented as regards the variation of the velocity potential on the plate at the instant of the impact which reveals the influence of the opening. The theory is extended to the computation of the total impulse exerted on the plate using pressure-impulse theory.

Keywords: Steep wave impact; pressure-impulse; elliptical harmonics; mixed boundary value problems; triple trigonestical series; integral equations.

\section{Introduction}

Knowing the effects of violent wave impact is crucial for the design of structures operating in, or close to the sea environment, such as harbour walls, moored or fixed offshore facilities and seagoing vessels. Violent breaking wave impact should be distinguished from the effects induced due to regular waves. Steep and breaking waves can exert forces many times greater than non-breaking waves or standing waves adjacent to a monolithic vertical harbour wall. In contrast to the latter which is a time varying continuous dynamic process that oscillates periodically, violent wave impact is a sudden and short-lived collision between a volume of water wave and a structure. The duration of the sudden impact is very short and induces huge hydrodynamic loads on the impacted structure. What it is interesting and vital in regards, is the study of the early stages of impact when the largest
hydrodynamic loading occurs.

Corresponding author: Tel. +30 210772 1105, Fax. +30 210772 1412, email: chatzi@naval.ntua.g
There are other issues as well that distinguish wave impact problems and make them particularly The wajor challenge that should be undertaken in the study of wave impact problems, mainly associated with three-dimensional (3D) formulations, is that they should be structured as boundary value problems with mixed conditions; so called Mixed Boundary Value Problems (MBVPs). An additional difficulty arises from the fact that in the general case of 3D wave impact, the impacted wetted region on the structure is one of the problem's unknowns and should be determined based on specific assumptions regarding the pressure and velocity of the liquid on the contact line where the free-surface meets the structure (Korobkin \& Scolan, 2006; Scolan \& Korobkin, 2001; Scolan, 2014). Nevertheless, we will not go deeper in that discussion as the physical model investigated in this paper determines explicitly the wetted region.

Judging from the published literature, wave impact has been studied theoretically much less than the class of problems associated with the entry of a body into initially static water - so called water-entry problems. In wave impact problems, the volume of liquid that impacts the structure has free-surfaces on two sides, the upper side and the steep wave front that hits the structure. In contrast, in water entry problems, which also lead to slamming, the single free-surface has simpler geometry. The more complicated shape and behaviour of the free-surfaces during the wave impact is harder to model than the water-entry.

Clearly, the proper formulation of a wave impact problem should account for the real 3D space. The difficulties associated with 3D descriptions and the solution methodologies that should be followed,

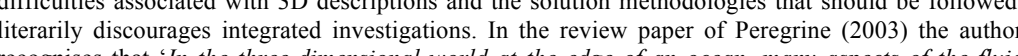
dynamics associated with analytical studies in wave impet problems two-dimensional (2D) formulations and approximations relevant to the geometric settings of the most violent impacts. These have reached a sufficient maturity and although they consider mainly impact on vertical walls, they introduce additional aspects that could influence the phenomenon. In this on vertical walls, they introduce additional aspects that could influence the phenomenon. In this breaking wave impact. Using the same method Wood \& Peregrine (2000) extended the previous work to study wave impact on a porous berm. The violent breaking wave impact was investigated in a to study wave impact on a porous berm. The
series of papers by Bullock et al. (2007) and Bredmose et al. (2009) \& (2015). Bullock et al. (2007) presented experimental results from breaking wave tests on vertical and inclined walls. Brendmose $e t$ al. (2009) considered the effect of the trapped air in the cavity formed by a breaking wave while in the last study of the same series, Brendmose et al. (2015) took into account possible aeration of the liquid. Cooker (2013) studied the interaction of breaking waves with permeable vertical walls. Experiments on the breaking wave impact on vertical walls were performed also by Cuomo et al. (2010). Overturning (plunging) breaking waves were generated by a sloping bed near the wall, while the width of the basin was sufficiently small, a fact that practically cancels $3 \mathrm{D}$ effects and sets the phenomenon in 2D. Examples of studies that rely on mancical methods to solve 2D problems of breaking wave on vertical walls are those due to Rafiee et al. (2015) and Carratelli et al. (2016). Both studies apply the method of Smoothed Particle Hydrodynamics (SPH) which appears to be more flexible than Computational Fluid Dynamics (CFD) solvers to impact problems.

An important simplification is to treat the face of the breaking wave as parallel to the wall at the instant of impact. For a vertical wall the wave front is considered completely vertical, ensuring maximum hydrodynamic load. In this context, Korobkin \& Malenica (2007) studied analytically the steep wave impact on an elastic wall, while recently Noar \& Greenhow (2015) applied the steep wave impact concept to rectangular geometries using pressure-impulse theory. Again, both studies were

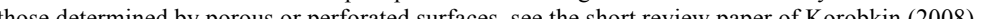

By contrast, there are few studies in 3D and they only employ numerical solvers. One method for called dam-break flow. That 
is, the wave generated by a volume of liquid which originally is confined by a barrier and released suddenly. In fact, the wave generated by the dam-break concept is the most realistic scenario of the steep wave. Examples of 3D studies on the subject are those due to Kleefsman et al. (2005) and Yang et al. (2010). The former study applied a Volume-of-Fluid (VOF) method to simulate the impact of a dam-break flow on perfectly rectangular bodies and Yang et al. (2010) used the unsteady Reynolds equations to simulate near-field dam-break flows and estimate the impact forces on obstacles. The cases considered resemble flood-like flows and they cannot be characterized as violent wave impact.

Dam-break flows have also been studied using Smoothed Particle Hydrodynamics (SHP) methodologies. Examples are the studies of Gómez-Gesteira and Dalrymple (2004) and Cummins $e t$ al. (2012) who examined the impact of a single wave, originating from a dam-break, with a tall coastal structure. In both studios, the structure was a vertical rectangular column. To the author's bes knowledge there have been no 3D studies, even using numerical methods, for more complicated convex geometries, such as circular cylinders. The main difficulty arises from the fact that the impacted wetted region is not known explicitly and must be treated as one of the problem's unknowns. Clearly, relevant difficulties are not encountered when the impacted structure is considered rectangular with the front face of the wave parallel to one of the sides of the box-shaped column. It is evident that in the concen case the region tis is hit by the wave is known in alvance. column's edges.

The present study is a contribution towards 3D approaches on wave impact problems. The physical model is a vertical plate subjected to a steep wave impact that propagates towards the plate with The opening is. The problem is complicated by assuning that the plate has a rectangular opening. rectangular opening through which liquid is disched, are varbe The complexity of the problem originates from the fact that two mixed boundary value problems (MBVP) should be coupered in two directions. To solve simultaneously both problems, the plate is assimilated by a degenerate cylinder with zero semi-minor axis. Having properly formulated one of the MBVP, the second (in the cylinder with zero semi-minor axis. Having properly formulated one of the MBVP, the second (in the
vertical direction) yields a one-dimensional MBVP involving triple trigonometrical series. It should be mentioned that in contrast to dual trigonometrical series, triple series of that kind are unfamiliar. The solution provided is based on the transformation of the triple trigonometrical series into triple series of integral equations. The solution method allows the derivation of analytical expressions for the velocity potential. Also, pressure-impulse theory is employed in order to calculate the total impulse on the plate.

The study is structured as follows: Section 2 formulates the hydrodynamic problem and describes the solution method employed to account for the mixed conditions in the horizontal direction. The solution method relies on the expansion of the velocity potential in elliptical harmonics, i.e. products of the radial and periodic Mathieu functions. Section 3 uses expansions in perturbations to formulate the triple trigononetrical series MBV. That problem is further analyzed in Section 4 while in Section 5 the associated MBVP is transformed into a MBVP involving integral equations. The method of solution relies on the reduction of the triple series into a dual series. That is achieved in Section 6 and Section 7 is dedicated to the solution of the dital series. Section 8 applies the pessure-impulse theory to calculate the total impulse exerted on the plate. Relevant computations are presented in followed by discussion. Finally, the conclusions of the study are provided in Section 9 .

\section{The hydrodynamic problem}

The steep wave propagates from $X>0$ towards the plate that is situated at $X=0$ (Fig. 1). The width of the plate is $2 L$ and the water depth is constant and equal to $H$. A Cartesian coordinate system is defined with its origin fixed on the plate at $X=0$, in the centre line of the plate at $Y=0$ and on the free-surface on $Z=0$. The $Z$-axis is pointing in the gravity direction and the flat bottom is situated on $Z=H$. The plate has a rectangular opening with its horizontal edges located at $Z=B$ and $Z=A$ from the free-surface. The opening allows the discharge of the liquid at the time of impact. The thickness of the plate at $B \leq Z \leq A$ and $Y= \pm L$ is considered negligible.

The liquid is assumed to be inviscid and incompressible and the flow irrotational allowing the employment of the linear potential theory. The velocity potential is denoted by $\Phi(X, Y, Z)$. We $\Phi=\phi V L, h=H / L, a=A / L$ and $b=B / L$. Accordingly, the flow will be described (in the nondimensional variables) through the following MBVP (see also Fig. 1).

$\nabla^{2} \phi=0,(x>0, \quad-\infty<y<\infty, 0<z<h)$

$\phi=0, \quad(x>0, \quad-\infty<y<\infty, z=0)$,

$\phi=0, \quad(x=0,|y|>1,0<z<h)$,

$\frac{\partial \phi}{\partial z}=0, \quad(x>0, \quad-\infty<y<\infty, \quad z=h)$

$\frac{\partial \phi}{\partial x}=1, \quad(x=0,|y|<1, \quad a<z<h)$

$\phi=0, \quad(x=0,|y|<1, \quad b<z<a)$,

$\frac{\partial \phi}{\partial x}=1, \quad(x=0,|y|<1,0<z<b)$

$\phi \rightarrow 0, x^{2}+y^{2} \rightarrow \infty$

The field equation under the employed assumptions for the flow is the Laplace's equation (2.1). The boundary conditons $(2.2),(2.3)$ and (2.6) follow from the lihearized dynanic conditions on the freesurfaces and the initial conditions that before the impact the fluid moves towards the plate with spatially uniform velocity $-\boldsymbol{V} t$. The function $\phi(x, y, z)$ is the sudden change in the velocity potential, consistent with the fluid remaining in contact with the plate after the impact. The condition (2.2) applies on the upper free-surface and the condition (2.3) refers to the front face of the steep wave, to the left and the right, beyond the impacted area. Condition (2.6) is the linearized condition on the rectangular opening through which the liquid is discharged. Also, (2.4) is the kinematic condition on the flat horizontal bottom, while (2.5) and (2.7) follow from the condition that the liquid hits the plate, does not penetrate it and wis wains in sliding contact with the impermeable upper and lower sections of the plate. Finally, $(2.8)$ is the far-field condition which indicates that the disturbance, owning to the impact, vanishes at infinity. The model equations (2.1)-(2.8) contain geometric parameters $a$ and $b$ to specify the position of $h<\pi / 2$ is also realistic. 


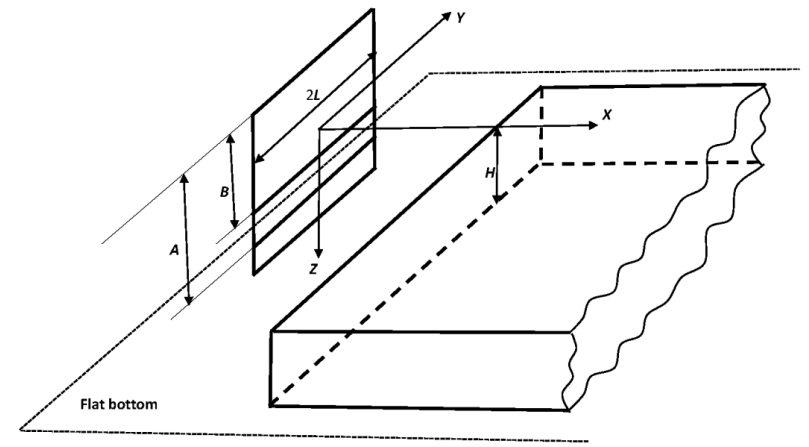

FIG. 1. The geometry of the impact problem.

To satisfy the system of (2.1)-(2.8), and to find an analytical solution to the linearized problem, we start by considering the following form for the velocity potential

$\phi(x, y, z)=\sum_{n=1}^{\infty} \phi_{n}(x, y) \sin \left(\lambda_{n} z\right)$

where $\lambda_{n}=(n-1 / 2) \pi / h$. Equation (2.9) satisfies the kinematic condition on the horizontal bottom (2.4) and the dynamic condition on the upper free-surface (2.2)

Substituting (2.9) into the Laplace equation (2.1) shows that the functions $\phi_{n}(x, y)$ satisfy the twodimensional Helmholtz equation

$\frac{\partial^{2} \phi_{n}}{\partial x^{2}}+\frac{\partial^{2} \phi_{n}}{\partial y^{2}}-\lambda_{n}^{2} \phi_{n}=0$

The solution for $\phi$ or equivalently $\phi_{n}$, should take into account the boundary condition (2.3) on the outer parts of the front face of the steep wave. Noting that a plate with finite dimensions can be effectively represented by an elliptical cylinder with zero semi-minor axis, we transform the Helmholtz equation using elliptical coordinates $(u, v)$, with

\section{$y=\bar{c} \cosh u \cos v$,}

$x=\bar{c} \sinh u \sin v$.

The elliptical coordinates $u=$ constant, $v=$ constant are orthogonally intersecting families of confocal ellipses and hyperbolae, respectively, while $\bar{c}$ is the half distance between the foci and is related with the semi-major and semi-minor axes of the elliptical cylinder $\alpha, \beta$ respectively by $\bar{c}=\sqrt{\alpha^{2}-\beta^{2}}$. The elliptical cylinder approximates a plate assuming $\beta \rightarrow 0$ and hence, in nondimensional form $\bar{c}=\alpha \rightarrow$ 1 . The surface of the plate is determined by $u_{0}=\tanh ^{-1}(\beta / \alpha) \rightarrow 0$.

Next, Moon \& Spencer (1971) show that in elliptical coordinates (2.10) is

$\frac{2}{\cosh 2 u-\cos 2 v}\left(\frac{\partial^{2} \phi_{n}}{\partial u^{2}}+\frac{\partial^{2} \phi_{n}}{\partial v^{2}}\right)-\lambda_{n}^{2} \phi_{n}=0$.
Assuming separable solutions, the component $\phi_{n}(u, v)$ that satisfies (2.13) is expanded in terms of elliptical harmonics as

$$
\begin{aligned}
\phi_{n}(u, v)=\sum_{m=0}^{\infty} \tilde{A}_{m}^{(n)} M c_{m}^{(1)}\left(u,-q_{n}\right) c e_{m}\left(v,-q_{n}\right)+\sum_{m=1}^{\infty} \tilde{B}_{m}^{(n)} M s_{m}^{(1)}\left(u,-q_{n}\right) s e_{m}\left(v,-q_{n}\right) \\
\quad+\sum_{m=0}^{\infty} \tilde{C}_{m}^{(n)} M c_{m}^{(3)}\left(u,-q_{n}\right) c e_{m}\left(v,-q_{n}\right) \\
\quad+\sum_{m=1}^{\infty} \tilde{S}_{m}^{(n)} M s_{m}^{(3)}\left(u,-q_{n}\right) s e_{m}\left(v,-q_{n}\right)
\end{aligned}
$$

where $c e$ and $s e$ denote the even and odd periodic Mathieu functions, while $M c$ and $M s$ are the even and odd modified (radial) Mathieu functions in the notation of Abramowitz \& Stegun (1970). The superscript labels (1) and (3) designate the kind of the radial Mathieu functions. Finally, $q_{n}=\lambda_{n}^{2} / 4$ and it is noted that the separation constant involved in the elliptical harmonics (the so-called Mathieu parameter), is negative.

The boundary conditions (2.3) and (2.8), expressed in terms of the expansions in elliptical harmonics $\phi_{n}(u, v)$ will read

$\phi_{n}(u,-\pi)=\phi_{n}(u, 0)=0$,

$\phi_{n}(u, v) \rightarrow 0, u \rightarrow \infty$

Of the radial functions present in the expression (2.14), only the Mathieu functions of the third kind satisfy the far-field condition (16). Accordingly the products that involve $M c_{m}^{(1)}$ and $M s_{m}^{(1)}$ are omitted. In addition, the conditions (2.15) are satisfied only by the odd periodic Mathieu functions $s e_{m}\left(v,-q_{n}\right)$. Lastly, it is remarked that the velocity potential is defined in the half plane $v \in[-\pi, 0]$ and as a result only the odd terms $m=2 r+1, r=0,1,2, \ldots$ should be retained. Hence, expression (2.14) is simply

$\phi_{n}(u, v)=\sum_{r=0}^{\infty} \tilde{S}_{2 r+1}^{(n)} M s_{2 r+1}^{(3)}\left(u,-q_{n}\right) s e_{2 r+1}\left(v,-q_{n}\right)$

where odd periodic Mathieu function can be written as

$s e_{2 r+1}\left(v,-q_{n}\right)=\sum_{k=0}^{\infty} B_{2 k+1}^{(2 r+1)} \sin [(2 k+1) v]$.

In (2.18) the constants $B_{2 k+1}^{(2 r+1)} \equiv B_{2 k+1}^{(2 r+1)}\left(-q_{n}\right)$ are the expansion coefficients of the odd periodic Mathieu functions $s e_{2 r+1}\left(v,-q_{n}\right)$.

The total velocity potential will read

$\phi(u, v, z)=\sum_{r=0}^{\infty} \sum_{n=1}^{\infty} \tilde{S}_{2 r+1}^{(n)} M s_{2 r+1}^{(3)}\left(u,-q_{n}\right) s e_{2 r+1}\left(v,-q_{n}\right) \sin \left(\lambda_{n} z\right)$

The unknown expansion coefficients $\tilde{S}_{2 r+1}^{(n)}$ are determined by applying the remaining boundary conditions (2.5)-(2.7). The tilde above $S$ will be dropped in the sequel. 
The boundary conditions (2.5)-(2.7) expressed in elliptical coordinates become

$\frac{\partial \phi}{\partial u}=\sin v, \quad(u=0, \quad-\pi<v<0,0<z<b)$,

$\phi=0,(u=0, \quad-\pi<v<0, b<z<a)$

$\frac{\partial \phi}{\partial u}=\sin v, \quad(u=0, \quad-\pi<v<0, a<z<h)$.

Next, we substitute the velocity potential from (2.19) into the boundary conditions $(2.20)-(2.22)$ to obtain

$\sum_{n=1}^{\infty} \sum_{r=0}^{\infty} S_{2 r+1}^{(n)} M s_{2 r+1}^{\prime(3)}\left(0,-q_{n}\right) s e_{2 r+1}\left(v,-q_{n}\right) \sin \left(\lambda_{n} z\right)$

$$
=\sin v,(0<z<b, a<z<h) \text {, }
$$

$\sum^{\infty} \sum^{\infty} S_{2 r+1}^{(n)} M s_{2 r+1}^{(3)}\left(0,-q_{n}\right) s e_{2 r+1}\left(v,-q_{n}\right) \sin \left(\lambda_{n} z\right)=0, \quad(b<z<a)$,

where the prime denotes differentiation with respect to the first argument. Both (2.23) and (2.24) are defined in the interval $-\pi<v<0$. It is noted that $M s_{2 r+1}^{(3)}\left(u,-q_{n}\right)$ is given by [Abramowitz \& Stegun, 1970; equations (20.8.9) and (20.8.11)]

$M s_{2 r+1}^{(3)}\left(u,-q_{n}\right)=\frac{2(-1)^{r+1}}{\pi} \sum_{k=0}^{\infty} \frac{A_{2 k+1}^{(2 r+1)}}{A_{1}^{(2 r+1)}}\left[I_{k}\left(u_{n}^{(1)}\right) K_{k+1}\left(u_{n}^{(2)}\right)+I_{k+1}\left(u_{n}^{(1)}\right) K_{k}\left(u_{n}^{(2)}\right)\right]$,

where $I$ and $K$ denote the modified Bessel functions of the first and the second kind respectively, $u_{n}^{(1)}=\sqrt{q_{n}} e^{-u}, u_{n}^{(2)}=\sqrt{q_{n}} e^{u}$ and $A_{2 k+1}^{(2 r+1)} \equiv A_{2 k+1}^{(2 r+1)}\left(q_{n}\right)$ are the expansion coefficients of the even periodic Mathieu functions $c e_{2 r+1}$. These are given by

$c e_{2 r+1}\left(v, q_{n}\right)=\sum_{k=0}^{\infty} A_{2 k+1}^{(2 r+1)} \cos [(2 k+1) v]$,

and $\quad s e_{2 r+1}\left(v,-q_{n}\right)=(-1)^{r} c e_{2 r+1}\left(\frac{\pi}{2}-v, q_{n}\right), \quad B_{2 k+1}^{(2 r+1)}\left(-q_{n}\right)=(-1)^{k-r} A_{2 k+1}^{(2 r+1)}\left(q_{n}\right)$, [Abramowitz \& Stegun, 1970; equation (20.8.4)]

Letting $u \rightarrow 0$ in (2.25) it can be shown that

$M S_{2 r+1}^{(3)}\left(0,-q_{n}\right) \rightarrow \frac{4(-1)^{r+1} c e_{2 r+1}\left(0, q_{n}\right)}{\pi \lambda_{n} A_{1}^{(2 r+1)}}$

For the derivation of (2.27) the Wronskian determinant for Bessel functions was used [Abramowitz \& Stegun, 1970; equation (9.6.15)]. The corresponding expression for the derivative of the radial Mathieu function as $u \rightarrow 0$ is more complicated and is given by
$M s_{2 r+1}^{(3)}\left(0,-q_{n}\right)$

$$
\begin{aligned}
& \rightarrow(-1)^{r+1} \frac{2 \sqrt{q_{n}}}{\pi A_{1}^{(2 r+1)}} \sum_{k=0}^{\infty} A_{2 k+1}^{(2 r+1)}\left[-I_{k}^{\prime}\left(\frac{\lambda_{n}}{2}\right) K_{k+1}\left(\frac{\lambda_{n}}{2}\right)\right. \\
& \left.+I_{k}\left(\frac{\lambda_{n}}{2}\right) K_{k+1}^{\prime}\left(\frac{\lambda_{n}}{2}\right)-I_{k+1}^{\prime}\left(\frac{\lambda_{n}}{2}\right) K_{k}\left(\frac{\lambda_{n}}{2}\right)+I_{k+1}\left(\frac{\lambda_{n}}{2}\right) K_{k}^{\prime}\left(\frac{\lambda_{n}}{2}\right)\right] .
\end{aligned}
$$

In the case of a solid rigid plate without the opening the body condition is described solely by (2.23) defined in the intervals $-\pi<v<0$ and $0<z<h$. Hence, employing orthogonality of the involved eigenfunctions, the expansion coefficients $S_{2 r+1}^{(n)}$ are

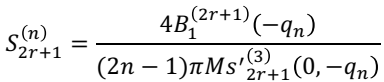

and the velocity potential $\phi(u, v, z)$, calculated exactly on the plate at $u=0$ from (2.19) is given by

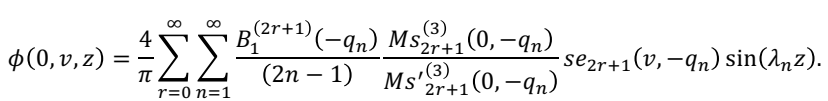

Equation (2.30) is valid unconditionally for any aspect ratio $h>0$. Also, (2.30) can provide the 2D strip theory solutions with vertical or horizontal strips assuming a very wide plate, $h \ll 1, q_{n} \rightarrow \infty$, or a very narrow plate, $h \gg 1, q_{n} \rightarrow 0$. Here we are interested in only the former case and therefore further analysis assuming $h \gg 1$ is omitted. Taking the asymptotics of the derivative of the radial Mathieu function for very large Mathieu parameters which implies taking the asymptotics of the Bessel functions (and their derivatives) for very large arguments [see also (2.28)] it can be shown that

$M s_{2 r+1}^{\prime(3)}\left(0,-q_{n}\right) \rightarrow \frac{4(-1)^{r} c e_{2 r+1}\left(0, q_{n}\right)}{\pi A_{1}^{(2 r+1)}}$.

Using (2.27) and (2.31), (2.30) becomes

$\phi(0, v, z)=-\frac{8 h}{\pi^{2}} \sum_{n=1}^{\infty} \frac{\sin [(2 n-1) \pi z(2 h)]}{(2 n-1)^{2}}\left[\sum_{r=0}^{\infty} B_{1}^{(2 r+1)}\left(-q_{n}\right) s e_{2 r+1}\left(v,-q_{n}\right)\right]$

Assuming that the term is square brackets equals an arbitrary function $f_{n}(v)$, then we are able to apply the orthogonality relation of $s e_{2 r+1}\left(v,-q_{n}\right)$ for $v \in[-\pi, 0]$. This gives

$\sum_{r=0}^{\infty} B_{1}^{(2 r+1)}\left(-q_{n}\right) \int_{-\pi}^{0} s e_{2 r+1}\left(v,-q_{n}\right) s e_{2 m+1}\left(v,-q_{n}\right) d v=\int_{-\pi}^{0} f_{n}(v) s e_{2 m+1}\left(v,-q_{n}\right) d v$.

The left hand side of (2.33) equals $\frac{\pi}{2} B_{1}^{(2 m+1)}\left(-q_{n}\right)$. Hence, by comparing with (2.18) the identity (2.33) holds if and only if $f_{n}(v)=\sin v$. The 2D solution is obtained by letting $v=-\frac{\pi}{2}$ and eventually (2.32) become

$\phi\left(0,-\frac{\pi}{2}, z\right)=\frac{8 h}{\pi^{2}} \sum_{n=1}^{\infty} \frac{\sin [(2 n-1) \pi z /(2 h)]}{(2 n-1)^{2}}$ 
Equation (2.34) is explicitly identical with the 2D strip theory solution $\phi^{(v)}(0, z)$ provided by King \& Needham (1994)

We now revert to the actual 3D problem, which has been reduced to (2.23) and (2.24). These equations represent a MBVP defined only in the vertical direction. In fact, (2.23) and (2.24) represent a triple trigonometrical series. Analytical solutions for relevant problems are feasible only if the unknown expansion coefficients that multiply the trigonometrical functions are the same. Here however, we treat a different, and more complicated case, as the coefficients of $\sin \left(\lambda_{n} z\right)$ in $(2.23)$ depend on the derivative of the radial Mathieu function, whilst those of (2.24) depend on the Mathieu function itself. To address properly this challenge a more sophisticated approach should be taken, as described in the next section.

\section{Expansion of the derivative of the radial Mathieu function in a series of} perturbations

Equation (2.28) is further elaborated using the series expansions of the modified Bessel functions that can be found in Abramowitz \& Stegun (1970) [equations (9.7.1) - (9.7.4)]. In particular it holds that

$$
\begin{aligned}
& I_{k}(z) \sim \frac{e^{z}}{\sqrt{2 \pi z}}\left[1-\frac{\mu-1}{8 z}+\frac{(\mu-1)(\mu-9)}{2 !(8 z)^{2}}-\frac{(\mu-1)(\mu-9)(\mu-25)}{3 !(8 z)^{3}}+O\left(1 / z^{4}\right)\right], \\
& K_{k}(z) \backsim \sqrt{\frac{\pi}{2 z}} e^{-z}\left[1+\frac{\mu-1}{8 z}+\frac{(\mu-1)(\mu-9)}{2 !(8 z)^{2}}+\frac{(\mu-1)(\mu-9)(\mu-25)}{3 !(8 z)^{3}}+O\left(1 / z^{4}\right)\right], \\
& I_{k}^{\prime}(z) \backsim \frac{e^{z}}{\sqrt{2 \pi z}}\left[1-\frac{\mu+3}{8 z}+\frac{(\mu-1)(\mu+15)}{2 !(8 z)^{2}}-\frac{(\mu-1)(\mu-9)(\mu+35)}{3 !(8 z)^{3}}+O\left(1 / z^{4}\right)\right], \\
& K_{k}^{\prime}(z) \backsim-\sqrt{\frac{\pi}{2 z}} e^{-z}\left[1+\frac{\mu+3}{8 z}+\frac{(\mu-1)(\mu+15)}{2 !(8 z)^{2}}+\frac{(\mu-1)(\mu-9)(\mu+35)}{3 !(8 z)^{3}}\right. \\
& \left.+O\left(1 / z^{4}\right)\right],
\end{aligned}
$$

where $\mu=4 k^{2}$. Substituting (3.1)-(3.4) into (2.28) and performing lengthy algebraic manipulations, it can be shown that the $O\left(1 / \lambda_{n}\right)$ term of the component within the square brackets of $(2.28)$ vanishes. Overall (2.28) can be written as

$M s_{2 m+1}^{\prime(3)}\left(0,-q_{n}\right)=-\lambda_{n} M s_{2 m+1}^{(3)}\left(0,-q_{n}\right)+\frac{(-1)^{m}}{8 \pi q_{n} A_{1}^{(2 m+1)}} \sum_{k=0}^{\infty}(2 k+1) A_{2 k+1}^{(2 m+1)}+O\left(\lambda_{n}^{-3}\right)$

Defining $\epsilon_{n}=\lambda_{n}^{-2}(3.5)$ can be written as

$M s_{2 m+1}^{(3)}\left(0,-q_{n}\right)=-\lambda_{n}^{-1} M s_{2 m+1}^{\prime(3)}\left(0,-q_{n}\right)+\epsilon_{n} \lambda_{n}^{-1} \Lambda_{2 m+1}^{(n)}+\cdots$

where we define

$\Lambda_{2 m+1}^{(n)}=\frac{(-1)^{m}}{2 \pi A_{1}^{(2 m+1)}} \sum_{k=0}^{\infty}(2 k+1) A_{2 k+1}^{(2 m+1)}$.
The second term in the right had side of (3.6) is negligible only if $\sqrt{q_{n}}$ is considered sufficiently large allowing the employment of the asymptotic formulae of the modified Bessel functions and their relatively wide plate we a cons if $O\left(1 / z^{3}\right)$ terms in (3.1) (3.4). The peth , in $O\left(1 / z^{3}\right)$ tens in (3.1)-(3.4). Che pet, if order to be able to en. ertheless, the re first one. That leads to the requirement $L>\frac{2}{\pi} H$.

The form obtained for the radial Mathieu function [see (3.6)] suggests taking the following expansion for the coefficients $S_{2 m+1}^{(n)}$ [see (2.23) and (2.24)]

$S_{2 m+1}^{(n)}=S_{2 m+1}^{(n),(0)}+\epsilon_{n} S_{2 m+1}^{(n),(1)}+\cdots$

Next, substituting (3.6) and (3.8) into (2.23) and (2.24) and equating like powers of $\epsilon_{n}$ we obtain the following systems at orders $O\left(\epsilon_{n}^{0}\right)$ and $O\left(\epsilon_{n}^{1}\right)$ :

Order $O\left(\epsilon_{n}^{0}\right)$ :

$\sum_{n=1}^{\infty} D_{n}^{(0)}(v) \sin \left(\lambda_{n} z\right)=\sin v, \quad(0<z<b, a<z<h)$,

$\sum_{n=1}^{\infty}\left(n-\frac{1}{2}\right)^{-1} D_{n}^{(0)}(v) \sin \left(\lambda_{n} z\right)=0,(b<z<a)$.

Order $O\left(\epsilon_{n}^{1}\right)$ :

$\sum_{n=1}^{\infty} D_{n}^{(1)}(v) \sin \left(\lambda_{n} z\right)=0, \quad(0<z<b, a<z<h)$

$\sum_{n=1}^{\infty}\left(n-\frac{1}{2}\right)^{-1} D_{n}^{(1)}(v) \sin \left(\lambda_{n} z\right)=G^{(1)}(v, z), \quad(b<z<a)$,

where

$D_{n}^{(j)}(v)=\epsilon_{n}^{j} \sum_{m=0}^{\infty} S_{2 m+1}^{(n),(j)} M s_{2 m+1}^{(3)}\left(0,-q_{n}\right) s e_{2 m+1}\left(v,-q_{n}\right), j=0,1$

$G^{(1)}(v, z)=\frac{h^{2}}{\pi^{2}} \sum_{n=1}^{\infty} \sum_{m=0}^{\infty}\left(n-\frac{1}{2}\right)^{-3}(-1)^{m} S_{2 m+1}^{(n),(0)} \Lambda_{2 m+1}^{(n)} c e_{2 m+1}\left(\frac{\pi}{2}-v, q_{n}\right) \sin \left(\lambda_{n} z\right)$.

The two orders of $\epsilon_{n}$ will be referred in the sequel as the leading order and the first-order. Clearly, the solution of the first-order problem (3.11)-(3.12) dictates the solution of the problem at leading order 
For the leading order problem it is observed that the MBVP of (3.9) and (3.10) suggests assuming that the unknown expansion coefficients $D_{n}^{(0)}(v)$ are directly proportional to $\sin v$, and be written as $D_{n}^{(0)}(v)=\bar{D}_{n}^{(0)} \sin v$, where $\bar{D}_{n}^{(0)}$ are constants. Therefore the solution of the associated triple trigonometrical series will provide sets of coefficients, not functions of $v$. Those functions are simply obtained by the direct multiplication of the leading order constant expansion coefficients by $\sin v$. Neverheless, here we have chosen to follow a global ho che will concern $D_{n}^{(0)}(v)$ as general functions of $v$.

\section{Mixed boundary value problems involving triple trigonometrical series}

Although mixed boundary value problems leading to dual trigonometrical series are well treated in the literature, there are only a few studies on triple trigonometrical series. The first solution to dual series was given by Shepherd (1938). Analytical solutions have been given by Tranter (1959), (1960a) and Fres). Srvastav (1963) showed that certain dual trigonometrical relations can be reduced to a solutions.

Studies approaching the analytic solution of triple trigonometrical series are scarce. For example the classic book of Sneddon (1966) has references only on dual series, the book of Fabrikant (1991) doesn't mention dual or triple trigonometrical series, while the last book on the subject by Duffy (2008), has only one specific example on sine series. Examples on triple trigonometrical series are the studies of Tranter (1969) and Kerr et al. (1994). Tranter (1969) showed that the solution of triple trigonometrical series can be sought by solving an equivalent system of three integral equations.
Tranter's (1969) work however is incomplete because although he considered both sine and cosine series involving harmonics $\sin [(n-1 / 2) \theta]$ and $\cos [(n-1 / 2) \theta], 0 \leq \theta \leq \pi$, he did not consider the case when the argument $(n-1 / 2)$ that multiplies the expansion coefficients is reversed. In fact, this is exactly the case that should be treated herein.

We start by considering the leading order problem of (3.9) and (3.10) and we employ the following transformations: $x=\pi z / h, b_{1}=\pi b / h$ and $a_{1}=\pi a / h$. Further, making use of the orthogonality of $\sin [(n-1 / 2) x]$, we approximate $\sin v$ by

$\sin v=\sin v \frac{2}{\pi} \sum_{n=1}^{\infty}(n-1 / 2)^{-1} \sin [(n-1 / 2) x]$.

We now define

$E_{n}^{(0)}(v)=D_{n}^{(0)}(v)-\frac{2}{\pi}(n-1 / 2)^{-1} \sin v$,

and (3.9) and (3.10) are recast into

$\sum^{\infty} E_{n}^{(0)}(v) \sin [(n-1 / 2) x]=0,\left(0<x<b_{1}, a_{1}<x<\pi\right)$

$\sum_{n=1}^{\infty}\left(n-\frac{1}{2}\right)^{-1} E_{n}^{(0)}(v) \sin [(n-1 / 2) x]=G^{(0)}(v, x), \quad\left(b_{1}<x<a_{1}\right)$,

$G^{(0)}(v, x)=-\frac{2}{\pi} \sin v \sum_{n=1}^{\infty} \frac{\sin [(n-1 / 2) x]}{(n-1 / 2)^{2}}$

We choose to use a uniform expression for both $G^{(0)}(v, x)$ and $G^{(1)}(v, x)$ writing them as

$G^{(j)}(v, x)=-\frac{2}{\pi} \sum_{n=1}^{\infty} W_{n}^{(j)}(v) \sin [(n-1 / 2) x], j=0,1$,

where

$W_{n}^{(0)}(v)=(n-1 / 2)^{2} \sin v$

and using (48)

$W_{n}^{(1)}(v)=-\frac{h^{2}}{2 \pi} \sum_{m=0}^{\infty}\left(n-\frac{1}{2}\right)^{-3}(-1)^{m} S_{2 m+1}^{(n),(0)} \Lambda_{2 m+1}^{(n)} c e_{2 m+1}\left(\frac{\pi}{2}-v, q_{n}\right)$

With these remarks we realize that the first-order problem is reduced to exactly the same form as the leading order problem, described through $(4,3)$ and $(4.4)$, after replacing the index $(0)$ by the index (1), using $E_{n}^{(1)}(v)=D_{n}^{(1)}(v)$ and taking $G^{(1)}(v, z) \equiv G^{(1)}(v, x)$ from (4.6), (4.8)

\section{Transformation of the trigonometrical series into a system of integral equations}

Following the work of Williams (1963), the idea of solving MBVPs involving triple trigonometrical series through transformation into integral equations was inspired by Tranter (1969). However, Tranter (1969) considered only cases where in the intermediate interval, here indicated by $b_{1}<x<$ $a_{1}$, the multipliers of trigonometrical functions (both sine and cosine) have the form $(n-1 / 2) E_{n}$. Although that seems insignificant, it literally introduces a major differentiation without allowing the employment of Tranter's (1969) method that was based on the Jacobi's expansion in a series of Bessel coefficients (Watson, 1944; p. 22).

To reform the triple trigonometrical series (4.3)-(4.4) into a system of triple integral equations we initial employ the transformation $u=\sin (x / 2) x=2 \sin ^{-1}(u)$ and we use the following useful relations that can be found in Gradshteyn \& Ryzhik (2007), p. 717 and 727

$\sin \left[(2 n-1) \sin ^{-1}(u)\right]=\sqrt{1-u^{2}} \int_{0}^{\infty} J_{2 n-1}(t) \sin (u t) d t$,

$\frac{1}{2 n-1} \sin \left[(2 n-1) \sin ^{-1}(u)\right]=\int_{0}^{\infty} t^{-1} J_{2 n-1}(t) \sin (u t) d t$.

Using (5.1) and (5.2), the system of (4.3) and (4.4) yields for $j=0,1$,

$\sum_{n=1}^{\infty} E_{n}^{(j)}(v) \int_{0}^{\infty} J_{2 n-1}(t) \sin (u t) d t=0, \quad\left(0<u<b_{2}, \quad a_{2}<u\right)$ 
$\sum_{n=1}^{\infty} E_{n}^{(j)}(v) \int_{0}^{\infty} t^{-1} J_{2 n-1}(t) \sin (u t) d t=\frac{1}{2} G^{(j)}\left[v, 2 \sin ^{-1}(u)\right], \quad\left(b_{2}<u<a_{2}\right)$,

where $a_{2}=\sin \left(a_{1} / 2\right)$ and $b_{2}=\sin \left(b_{1} / 2\right)$

Accordingly, using [Abramowitz \& Stegun, 1970; equations (10.1.1) and (10.1.11)]

$\sin (u t)=\sqrt{\frac{\pi u t}{2}} J_{\mu}(u t), \quad \mu=\frac{1}{2}$

and assuming

$E^{(j)}(v, t)=\sum_{n=1}^{\infty} E_{n}^{(j)}(v) J_{2 n-1}(t)$,

the system of (5.3) and (5.4) finally becomes

$\int_{0}^{\infty} t^{1 / 2} E^{(j)}(v, t) J_{\mu}(u t) d t=0, \quad\left(0<u<b_{2}, \quad a_{2}<u\right)$,

$\int_{0}^{\infty} t^{-1 / 2} E^{(j)}(v, t) J_{\mu}(u t) d t=\frac{1}{2} \sqrt{\frac{2}{\pi u}} G^{(j)}\left[v, 2 \sin ^{-1}(u)\right], \quad\left(b_{2}<u<a_{2}\right)$.

The system of (5.7) and (5.8) is usually referred in the literature as system of Titchmarsh type rsh, 1948)

\section{Solution of the triple integral equations}

Systems alike (5.7) and (5.8) are usually processed by attempting to satisfy by default one of the involved equations in a specific interval and substituting subsequently the assumed solution to the remaining relations. The procedure that is followed in the present exploits the following integral relation

$\int_{0}^{\infty} t^{-p} J_{\mu+2 n-1+p}\left(a_{2} t\right) J_{\mu}(u t) d t=0, \quad\left(a_{2}<u\right)$,

for $p= \pm \frac{1}{2}$ and $n=1,2,3, \ldots$.

For $u<a_{2}$ the integral yields nonzero values and in particular

$\int_{0}^{\infty} t^{p} J_{\mu+2 n-1+p}\left(a_{2} t\right) J_{\mu}(u t) d t$

$$
=\frac{2^{p} u^{\mu} \Gamma(n+\mu+p)}{a_{2}^{\mu+1+p} \Gamma(\mu+1) \Gamma(n)}{ }_{2} F_{1}\left(n+\mu+p,-n+1 ; \mu+1 ; \frac{u^{2}}{a_{2}^{2}}\right),
$$

$\int_{0}^{\infty} t^{-p} J_{\mu+2 n-1+p}\left(a_{2} t\right) J_{\mu}(u t) d t$

$$
\begin{aligned}
& =\frac{2^{-p} u^{\mu} \Gamma(n+\mu)}{a_{2}^{\mu+1-p} \Gamma(\mu+1) \Gamma(n+p)}{ }_{2} F_{1}\left(n+\mu,-n+1-p ; \mu+1 ; \frac{u^{2}}{a_{2}^{2}}\right) \\
& =\frac{2^{-p} u^{\mu} \Gamma(n+\mu)\left(1-\frac{u^{2}}{a_{2}^{2}}\right)^{p}}{a_{2}^{\mu+1-p} \Gamma(\mu+1) \Gamma(n+p)}{ }_{2} F_{1}\left(-n+1, n+\mu+p ; \mu+1 ; \frac{u^{2}}{a_{2}^{2}}\right),
\end{aligned}
$$

where ${ }_{2} F_{1}$ denotes the hypergeometric function. The integral that appears in (6.2)-(6.3) is known as the Sonine-Schafheitlin integral [Gradshteyn \& Ryzhik, 2007; p. 683, equations $(6.574 .1)$ and (6.574.3); Watson, 1944, p. 401, equation (2): Tranter, 1969] The convergence of the integrals in (6.2)-(6.3) requires that $\operatorname{Re}(\mu)>-1$ when $p=\frac{1}{2}$ and $\operatorname{Re}(\mu)>-\frac{1}{2}$ when $p=-\frac{1}{2}$. These conditions are satisfied by default herein as $\mu=\frac{1}{2}$.

Accordingly we assume the following form for the unknown function $E^{(j)}(v, t)$

$E^{(j)}(v, t)=\sum_{n=1}^{\infty} d_{n}^{(j)}(v) J_{\mu+2 n-1+p}\left(a_{2} t\right)$

while we let $p=-\frac{1}{2}$. Hence, substituting (6.4) into (5.7) and rearranging the summation and the integration the following is obtained

$\sum_{n=1}^{\infty} d_{n}^{(j)}(v) \int_{0}^{\infty} t^{-p} J_{\mu+2 n-1+p}\left(a_{2} t\right) J_{\mu}(u t) d t=0$,

which holds true for $a_{2}<u$ due to (6.1). Thus, we have satisfied one of the three integral equations and in particular the one which should be valid for $a_{2}<u$. Accordingly, (6.4) is substituted into the remaining two integral equations of (5.7) and (5.8). By exploiting (6.2) and (6.3) for $u<a_{2}$ and performing some brief algebraic calculations the following are derived

$\sum_{n=1}^{\infty}(n-1 / 2) d_{n}^{(j)}(v){ }_{2} F_{1}\left[n+\frac{1}{2}, 2-\left(n+\frac{1}{2}\right) ; \frac{3}{2} ; \sin ^{2} \theta\right]=0, \quad\left(0<\theta<\frac{b_{2}}{a_{2}}\right)$,

$\sum_{n=1}^{\infty} d_{n}^{(j)}(v){ }_{2} F_{1}\left[n, 1-n ; \frac{3}{2} ; \sin ^{2} \theta\right]=\frac{1}{2 \sin \theta} G^{(j)}\left[v, 2 \sin ^{-1}\left(a_{2} \sin \theta\right)\right], \quad\left(\frac{b_{2}}{a_{2}}<\theta<\frac{\pi}{2}\right)$,

where we used $u=a_{2} \sin \theta$. Equations (6.6) and (6.7) can be simplified significantly by expressing the hypergeometric functions in terms of sinusoidal harmonics [see equations (15.1.15) and (15.1.16) in Abramowitz \& Stegun 1970]. In order to increase the interval, in which the independent variable is $\sum_{n=1}^{\infty}(n-1 / 2) c_{n}^{(j)}(v) \sin [(n-1 / 2) \vartheta]=0=0, \quad\left(0<\vartheta<d^{*}\right)$, 
$\sum_{n=1}^{\infty} c_{n}^{(j)}(v) \sin [(n-1 / 2) \vartheta]=G^{(j)}\left\{v, 2 \sin ^{-1}\left[a_{2} \sin (\vartheta / 2)\right]\right\}, \quad\left(d^{*}<\vartheta<\pi\right)$,

where $d^{*}=2 b_{2} / a_{2}$.

\section{Solution of the dual trigonometrical series}

7.1 Computation of the expansion coefficients of the dual trigonometrical series

Equations (6.8) and (6.9) form a one-dimensional MBVP that involves dual trigonometrical series. Systems of that kind are well treated in the literature and there have been several authors who provided proper solutions. For a summary the reader is referred to the classical book of Sneddon (1966). However, it should be mentioned that the suggested solutions were derived without being complemented by numerical computations. The solution provided in Sneddon (1966) for instance, although accurate, does not allow efficient numerical performance as it requires the computation of the derivative of an integral for which no analytical solution exists and accordingly the only feasible way is to elaborate numerically. This type of complications however are prone to numerical inaccuracies and accordingly the system of (6.8) and (6.9) is processed further using the methodology suggested by Tranter (1969).

By employing the transformations

$$
\gamma=\pi-d^{*} ; \vartheta=\pi-y ;(-1)^{n-1} c_{n}^{(j)}(v)(n-1 / 2)=s_{n}^{(j)}(v)
$$

the system of (6.8) and (6.9) yields

$\sum_{n=0}^{\infty} \frac{s_{n}^{(j)}(v)}{2 n+1} \cos [(n+1 / 2) y]=\frac{1}{2} G^{(j)}\left\{v, 2 \sin ^{-1}\left[a_{2} \cos (y / 2)\right]\right\}, \quad(0<y<\gamma)$,

$\sum_{n=0}^{\infty} s_{n}^{(j)}(v) \cos [(n+1 / 2) y]=0, \quad(\gamma<y<\pi)$.

The system of (7.1) and (7.2) has been considered by Tranter (1960b) who found an appropriate way to employ Mehler's integrals (Magnus \& Oberhettinger, 1949; p. 52). The solution provided (adapted in the present system) relies on the following recurrence relation for the computation of the expansion coefficients $s_{n}^{(j)}(v)$

$s_{n}^{(j)}(v)=s_{0}^{(j)}(v) P_{n}(\cos \gamma)-\int_{0}^{\gamma} F^{(j)}(v, t) P_{n}^{\prime}(\cos t) \sin t d t, n=1,2,3, \ldots$

The remaining coefficient $s_{0}^{(j)}(v)$ is found by substitution of (7.3) into (7.1) for any value in the corresponding interval. For simplicity, this is chosen to be $y=0$. Hence

$s_{0}^{(j)}(v)=\left\{\frac{1}{2} G^{(j)}\left[v, 2 \sin ^{-1}\left(a_{2}\right)\right]+\sum_{n=1}^{\infty}(2 n+1)^{-1} \int_{0}^{\gamma} F^{(j)}(v, t) P_{n}^{\prime}(\cos t) \sin t d t\right\}$

$$
/\left\{1+\sum_{n=1}^{\infty}(2 n+1)^{-1} P_{n}(\cos \gamma)\right\},
$$

and the coefficients $s_{n}^{(j)}(v), n=1,2,3, \ldots$ are obtained by the recurrence relation $(7.3)$

In (7.3)-(7.4), $P_{n}$ denotes the Legendre polynomial with degree $n$ and the prime denotes differentiation with respect to the argument. The derivative of the Legendre polynomials can be determined by the summation theorem (Gradshteyn \& Ryzhik, 2007, p. 986)

$P_{n}^{\prime}(\tau)=\sum_{k=0}^{\left[\frac{n-1}{2}\right]}(2 n-4 k-1) P_{n-2 k-1}(\tau)$,

$\tau P_{n}^{\prime}(\tau)-n P_{n}(\tau)=\sum_{k=0}^{\left[\frac{n-2}{2}\right]}(2 n-4 k-3) P_{n-2 k-2}(\tau)$,

where $\tau$ can be any complex number.

The function $F^{(j)}(v, t)$ involved in (7.3)-(7.4) is given by the elliptic integral

$F^{(j)}(v, t)=\frac{\sqrt{2}}{\pi} \int_{0}^{t} \frac{d G^{(j)}\left\{v, 2 \sin ^{-1}\left[a_{2} \cos (y / 2)\right]\right\} / d y}{\sqrt{\cos y-\cos t}} \sin y d y$

or by evaluating the derivative

$F^{(j)}(v, t)=$

$\frac{2 \sqrt{2}}{\pi^{2}} a_{2} \sum_{n=1}^{\infty}\left(n-\frac{1}{2}\right) W_{n}^{(j)}(v) \int_{0}^{t} \frac{\sin y \sin (y / 2) \cos \left\{(2 n-1) \sin ^{-1}\left[a_{2} \cos (y / 2)\right]\right\}}{\sqrt{1-a_{2}^{2} \cos ^{2}(y / 2)} \sqrt{\cos y-\cos t}} d y$.

Although, the integral in (7.8) looks relatively complicated, does not pose major difficulties in its numerical computation. In any event, it should be treated as an improper integral. As already mentioned, the solution at order $O\left(\epsilon_{n}^{1}\right)$ requires the solution at the leading order $O\left(\epsilon_{n}^{0}\right)$. In other words, one must obtain the original expansion coefficients $S_{2 m+1}^{(n),(0)}$. Therefore the reversed procedure must be applied all the way to the very beginning to calculate the original expansion coefficients $S_{2 m+1}^{(n),(0)}$. Nevertheless, this is not a simple straightforward process.

\subsection{The reverse procedure}

The reverse procedure could be applied at both orders $O\left(\epsilon_{n}^{0}\right)$ and $O\left(\epsilon_{n}^{1}\right)$ to obtain the original coefficients of the Mathieu series expansions $S_{2 m+1}^{(n),(j)}, j=0,1$. Nevertheless, as it is discussed in the sequel, the computation of the leading order potential can be obtained directly through $s_{n}^{(0)}(v)$, without the need to compute $S_{2 m+1}^{(n),(0)}$. The latter however are required for the triple trigonometrical series problem at $O\left(\epsilon_{n}^{1}\right)$ due to the form of $G^{(1)}(v, x)$ given by (4.6) and (4.8).

The reverse procedure requires to start from $s_{n}^{(j)}(v)$ and calculate sequentially $c_{n}^{(j)}(v), d_{n}^{(j)}(v)$, $E_{n}^{(j)}(v), D_{n}^{(j)}(v)$ and finally $S_{2 m+1}^{(n),(j)}$ through (3.13). Let us assume for the moment that we have been able to obtain $D_{n}^{(j)}(v)$. Then the derivation of $S_{2 m+1}^{(n),(j)}$ requires the employment of the orthogonality relation satisfied by the periodic Mathieu functions in (3.13). It is noted that $s e_{2 m+1}\left(v,-q_{n}\right)$ (as well
as the even periodic Mathieu functions) are orthogonal in both $v \in[-\pi, 0]$ and $v \in[0,2 \pi]$. The 
orthogonality constants are equal to $\pi / 2$ and $\pi$ respectively [Abramowitz \& Stegun, 1970; equation (20.5.3)]. Thus from (3.13) one gets for $j=0,1$,

$$
\begin{aligned}
S_{2 m+1}^{(n),(j)}=\frac{1}{\pi \epsilon_{n}^{j} M s_{2 m+1}^{\prime(3)}\left(0,-q_{n}\right)} \int_{0}^{2 \pi} D_{n}^{(j)}(v) s e_{2 m+1}\left(v,-q_{n}\right) d v \\
\quad=\frac{(-1)^{m}}{\pi \epsilon_{n}^{j} M s_{2 m+1}^{\prime(3)}\left(0,-q_{n}\right)} \int_{0}^{2 \pi} D_{n}^{(j)}(v) c e_{2 m+1}\left(\pi / 2-v,-q_{n}\right) d v .
\end{aligned}
$$

As already remarked in Section 3 the leading order coefficients $D_{n}^{(0)}(v)$ [as well as all the leading order coefficients defined in the present: $\left.s_{n}^{(0)}(v), c_{n}^{(0)}(v), d_{n}^{(0)}(v), E_{n}^{(0)}(v)\right]$ are directly proportional to $\sin v$ and can be written as $D_{n}^{(0)}(v)=\bar{D}_{n}^{(0)} \sin v$, where $\bar{D}_{n}^{(0)}$ are constants and they are literally the coefficients calculated by the outlined procedure. Hence it is easily deduced that

$S_{2 m+1}^{(n),(0)}=\frac{(-1)^{m} A_{1}^{(2 m+1)}\left(q_{n}\right) \bar{D}_{n}^{(0)}}{M s_{2 m+1}^{\prime(3)}\left(0,-q_{n}\right)}$.

The problem in the reversed procedure originates from the transformation of the trigonometrical series into integral equations. The turning point is (5.6) which together with (6.4) provide the identity

$\sum_{n=1}^{\infty} E_{n}^{(j)}(v) J_{2 n-1}(t)=\sum_{n=1}^{\infty} d_{n}^{(j)}(v) J_{\mu+2 n-1+p}\left(a_{2} t\right)$.

Assuming that the last coefficients we have calculated are those of the dual trigonometrical series (7.1) and (7.2), then we can easily see that $d_{n}^{(j)}(v)=(-1)^{n-1} s_{n}^{(j)}(v)$. However, there is no straightforward procedure to obtain $E_{n}^{(j)}(v)$ through $d_{n}^{(j)}(v)$ using (7.11) and apparently the latter must be processed further.

Thus, we use again $u=\sin (x / 2), x=2 \sin ^{-1}(u), 0 \leq u \leq 1,0 \leq x \leq \pi$ and we multiply both sides of $(7.11)$ by $\sin (u t)$. Accordingly we integrate in the semi-infinite interval $[0, \infty)$ to obtain

$\sum_{n=1}^{\infty} E_{n}^{(j)}(v) \int_{0}^{\infty} J_{2 n-1}(t) \sin (u t) d t=\sum_{n=1}^{\infty} d_{n}^{(j)}(v) \int_{0}^{\infty} J_{2 n-1}\left(a_{2} t\right) \sin (u t)$.

Note that $\mu=\frac{1}{2}$ and $p=-\frac{1}{2}$. We define $J_{2 n-1}(u)=\int_{0}^{\infty} J_{2 n-1}\left(a_{2} t\right) \sin (u t) d t$ which is calculated by [Gradshteyn \& Ryzhik, 2007; equation (6.6.71), p.17]

$J_{2 n-1}(u)= \begin{cases}\frac{\sin \left[(2 n-1) \sin ^{-1}\left(\frac{u}{a_{2}}\right)\right]}{\sqrt{a_{2}^{2}-u^{2}}}, & u<a_{2} \\ 0, & u=a_{2} \\ \frac{a_{2}^{2 n-1} \cos [(n-1 / 2) \pi]}{\sqrt{u^{2}-a_{2}^{2}}\left(u+\sqrt{u^{2}-a_{2}^{2}}\right)^{2 n-1}}, & u>a_{2}\end{cases}$

Equation (7.13) implies that $\jmath_{2 n-1}(u)=0$ for $u \geq a_{2}$. It can also be employed using $a_{2}=1$. Therefore (7.11) yields $\sum_{n=1}^{\infty} E_{n}^{(j)}(v) \sin [(n-1 / 2) x]=\sum_{n=1}^{\infty} d_{n}^{(j)}(v) \frac{\sin \left\{(2 n-1) \sin ^{-1}\left[\frac{\sin (x / 2)}{a_{2}}\right]\right\} \cos (x / 2)}{\sqrt{a_{2}^{2}-\sin ^{2}(x / 2)}}$.

The derivation of (7.14) allows the employment of the orthogonality relation of $\sin [(n-1 / 2) x]$ in $0 \leq x \leq \pi$. Therefore the expansion coefficients $E_{n}^{(j)}(v)$ will yield from $d_{n}^{(j)}(v)$ through

$E_{n}^{(j)}(v)$

$=\frac{2}{\pi} \sum_{n=1}^{\infty} d_{n}^{(j)}(v) \int_{0}^{2 \sin ^{-1}\left(a_{2}\right)} \frac{\sin \left\{(2 n-1) \sin ^{-1}\left[\frac{\sin (x / 2)}{a_{2}}\right]\right\} \cos (x / 2) \sin \left[\left(n-\frac{1}{2}\right) x\right]}{\sqrt{a_{2}^{2}-\sin ^{2}(x / 2)}} d x$

Having calculated $E_{n}^{(j)}(v)$, the coefficients $D_{n}^{(0)}(v)$ will be given by $(4.2), D_{n}^{(1)}(v)=E_{n}^{(1)}(v)$, while the original expansion coefficients will be obtained using (3.13).

\section{Velocity potential and pressure-impulse}

\subsection{The velocity potential}

The method of perturbations suggests taking the following form for the total velocity potential, calculated exactly on the surface of the plate for $u=0$

$\phi(0, v, z)=\phi^{(0)}(0, v, z)+\phi^{(1)}(0, v, z)+\cdots$

Accordingly, use of (2.19), (3.6) and (3.8) requires that

$\phi^{(0)}(0, v, z)=-\sum_{m=0}^{\infty} \sum_{n=1}^{\infty} \lambda_{n}^{-1} S_{2 m+1}^{(n),(0)} M s_{2 m+1}^{\prime(3)}\left(0,-q_{n}\right) s e_{2 m+1}\left(v,-q_{n}\right) \sin \left(\lambda_{n} z\right)$,

$\phi^{(1)}(0, v, z)=$

$-\sum_{m=0}^{\infty} \sum_{n=1}^{\infty} \epsilon_{n} \lambda_{n}^{-1}\left[S_{2 m+1}^{(n),(1)} M s_{2 m+1}^{\prime(3)}\left(0,-q_{n}\right)-S_{2 m+1}^{(n),(0)} \Lambda_{2 m+1}^{(n)}\right] s e_{2 m+1}\left(v,-q_{n}\right) \sin \left(\lambda_{n} z\right)$.

The above are valid in the intervals $0<z<b, a<z<h$, while the potentials should be zero in the intermediate interval $b<z<a$. As a means for validating the present complicated method, we should verify that the potentials are indeed zero at the limiting points $z=a, z=b$. The curves of the potentials should be smooth and they should 'close' at the above mentioned limiting points.

The numerical implementation of the theory outlined in the present requires some attention as regards the value of the aspect ratio $h$ which in any case should be smaller than $\frac{\pi}{2}$. This can be traced back to the fact that the derivative of the radial Mathieu function was expanded in a series of perturbations and we retained only the leading order and the first-order terms of the series [see (3.6)]. Therefore for $1<h<\frac{\pi}{2}$ additional terms in the series of perturbations might be required. Here, we present numerical results for two sufficiently small aspect ratios, $h=0.5$ and $h=0.8$ which accordingly ensure that all $\epsilon_{n}, n=1,2,3 \ldots$ are sufficiently small allowing the approximation of the problem's parameters by the two only terms of the perturbation expansion.

It is frequently stated that $3 \mathrm{D}$ effects in $3 \mathrm{D}$ impact problems are important close to the contact line between the body and the liquid. In the present case, it is anticipated that 3D effects will be important 

close to the edges of the plate at $y= \pm 1$. Relevant allegations are usually provided without arises is whether the 3D effects are indeed important close to the plate's edges and literarily insignificant in the plate's vertical centreline. Also, given the fact that the potential is zero at the plate's edges, an additional question concerns the sections of the plate in which 3D effects are significant

The solution provided by (2.34) is easily reproduced by the leading order potential (8.2) (for $y=0 \mathrm{o}$ $v=-\pi / 2$ ) as shown in Fig. 2. To obtain the results of (2.34) through (8.2) we assumed an infinitesimal opening very close to the top of the plate, near the upper free-surface. Also, Fig. 2 depicts the velocity potential in the middle of the plate for a very small open area that was chosen to exist near the half of the plate's impacted height. The results are extremely interesting as they imply that if we allow the discharge of the liquid through a very small window, the velocity potential, and accordingly the pressure-impulse, on the two solid subsections are reduced substantially. The reason is that the velocity potential is forced to become zero at the horizontal edges of the open area. This finding has obvious significance for the design of coastal and offshore structures.

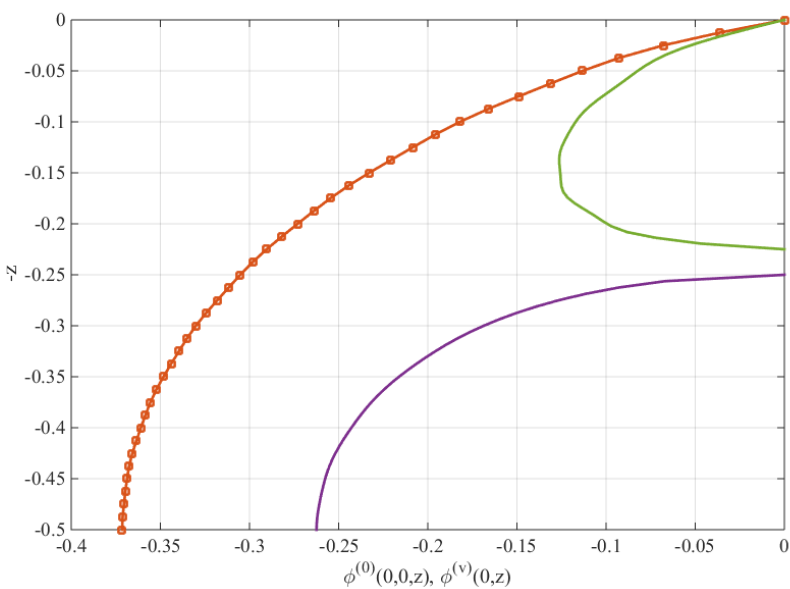

FIG. 2 The 2D strip theory solution (continuous curves) compared with 3D solution (symbols): $h=$ $H / L=0.5$. For solving the MBVP it was assumed $a / h=0.001, b / h=0.0001$. The second case to a wave impact on a plate with an open area situted in $0.45 h<z<0.5 h$

Figs. 3 and 4 show the behaviour of the velocity potential at the leading order and at the plate's centre line $y=0$, for a constant upper and lower edge respectively and increasing open area. The $2 \mathrm{D}$ solution (2.34) is also shown as a reference. At the lower part of the plate the potential obtains its maximum at the bottom and progressively decreases to zero at the first horizontal edge from the the maximum vast, at the upper part of the plate the potential exhibits a bow that the increase of the opening leads to a drastic decrease of the of the upper part. Both figurituses of the velocity potential. In the part of the plate that maintains a constant height, the decrease of the potential appears not to be influenced by the surface of the opening.
The cases considered in Figs. 3 and 4 correspond explicitly to the middle of the plate. The 3D potential varies transversely (along $y$, or $v$ ) until it becomes zero at the plate's lateral edges. In fact the leading order potential $\phi^{(0)}(0, y, z)$ varies proportionally with $\sin v$. This is better shown in the contour plots of Figs. 5-8 where the opening in which the potential is zero, is ims bediown in the contour plots shown in the sequel depict the half-width of the plate due to symmetry. The axes lengths correspond to the aspect ratio $h$ being considered. As far as the leading order potential is concerned, the $3 \mathrm{D}$ effects are explicitly described by the sinusoidal reduction from $v=-\pi / 2$ to $v=0$ (and $v=$ $-\pi$ ). The pairs of Figs. 5-6 and 7-8 show that, overall, the magnitude of the velocity potential $-\pi)$. Thes for increasing aspect ratio $h$ even though the normalized height and the position of the opening remain the same. The maxima of the leading order potential occur along the centreline of the contact region for $y=0$. On the lower section the maxima are detected on the bottom, while on the upper section the strongest effect occurs close to its middle (with a very small shift towards the bottom)

We now discuss the leading order potential computed for several cases. From figs. 5-8 we conclude that reducing the width of the gap increases the local maxima in potental on the upper and the lower the edges of the plate section. These consequenc gap near its upper and lower boundaries. Comparing Figs. 5, 7 and 6,8 it is easily deduced that allowing larger volume of liquid to pass through the opening on the plate, results in reduced impact effects, quantified by the velocity potential. The results due to the impact are mitigated not only because the contact region is redyced, but also becense An additional characteristic of the leading order potential, which should be highlighted, is related to is

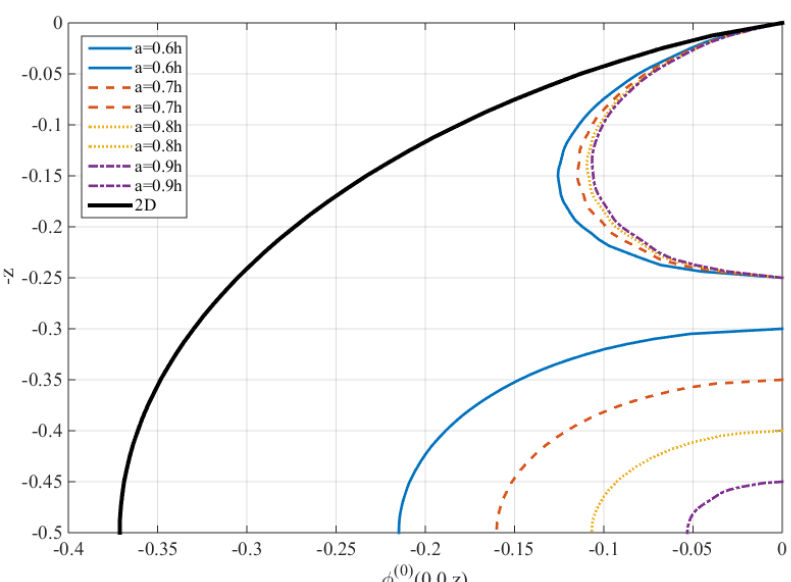

FIG. 3 The leading order potential $\phi^{(0)}(0,0, z)$ for a fixed upper edge $b / h=0.5$ and variable position of the lower edge $a / h$. The aspect ratio of the plate is $h=H / L=0.5$. 


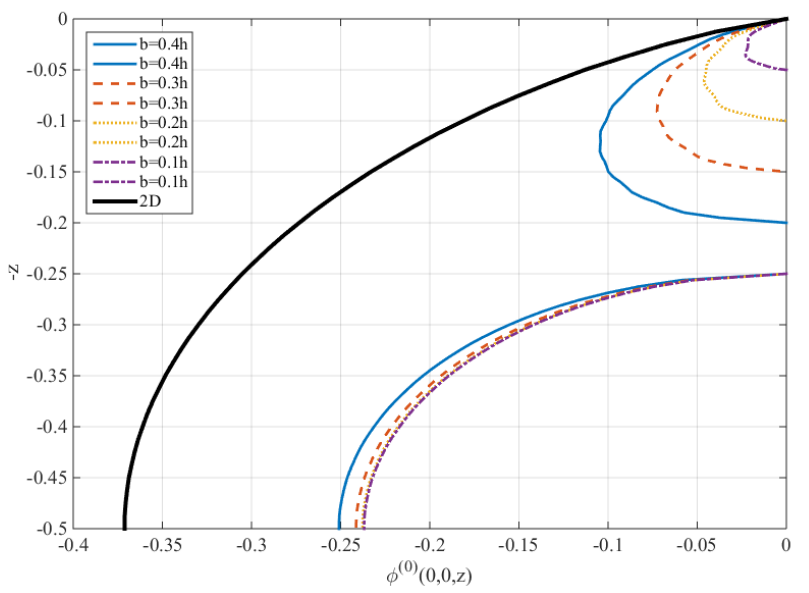

FIG. 4 The leading order potential $\phi^{(0)}(0,0, z)$ for a fixed lower edge $a / h=0.5$ and variable position of the upper edge $b / h$. The aspect ratio of the plate is $h=H / L=0.5$.

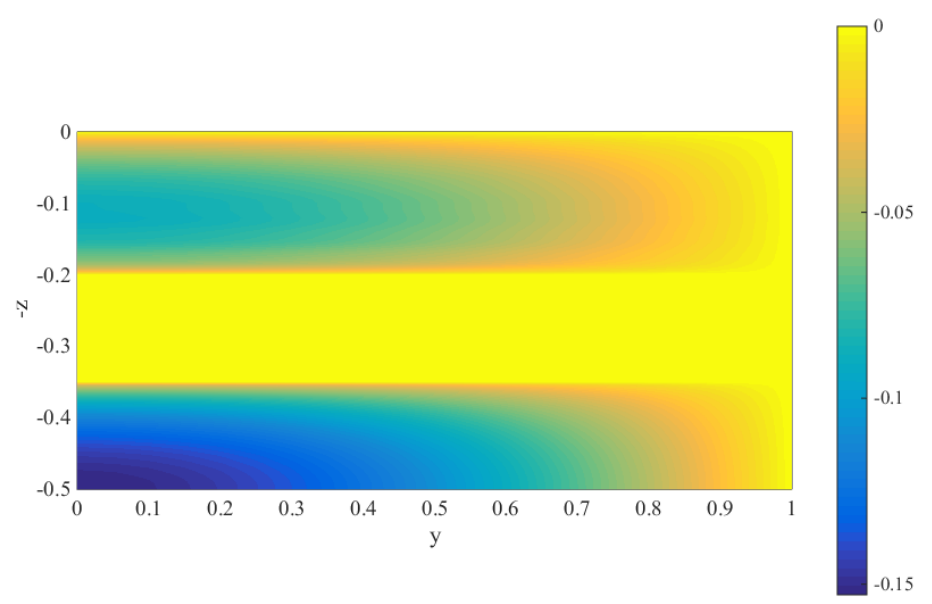

FIG. 5 The leading order potential $\phi^{(0)}(0, y, z)$ for lower edge $a / h=0.7$ and upper edge $b / h=0.4$. The aspect ratio of the plate is $h=H / L=0.5$.

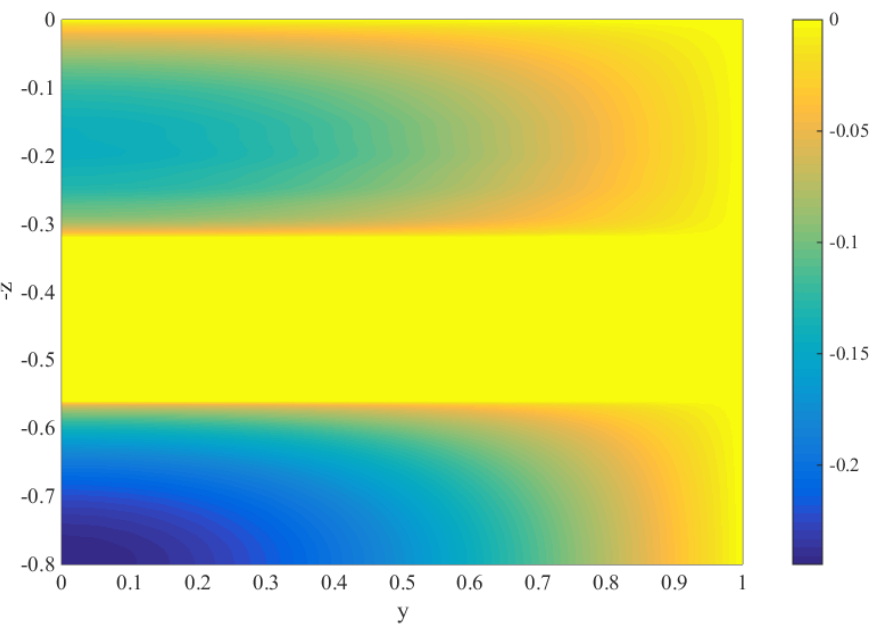

FIG. 6 The leading order potential $\phi^{(0)}(0, y, z)$ for lower edge $a / h=0.7$ and upper edge $b / h=0.4$. The aspect ratio of the plate is $h=H / L=0.8$.

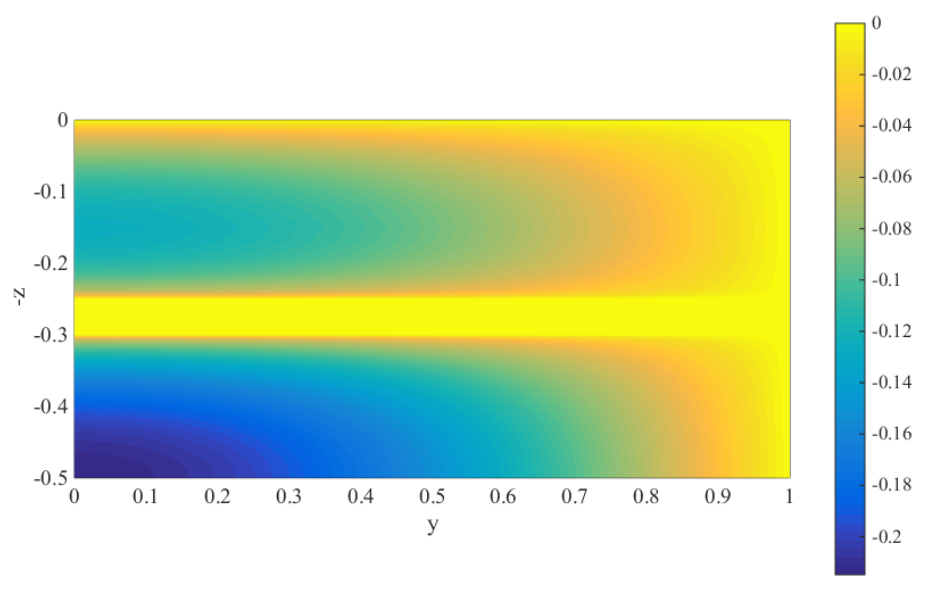

FIG. 7 The leading order potential $\phi^{(0)}(0, y, z)$ for lower edge $a / h=0.6$ and upper edge $b / h=0.5$. The aspect ratio of the plate is $h=H / L=0.5$. 


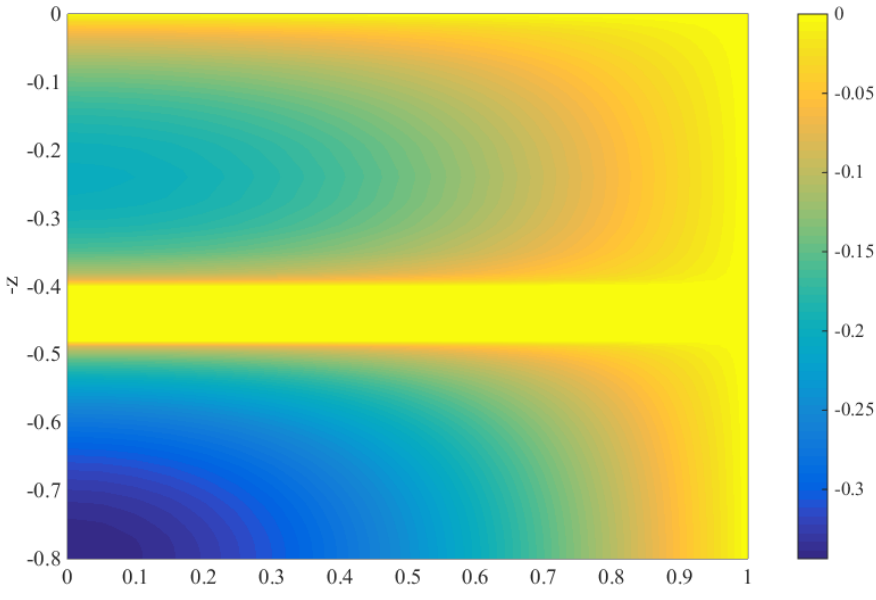

FIG. 8 The leading order potential $\phi^{(0)}(0, y, z)$ for lower edge $a / h=0.6$ and upper edge $b / h=0.5$. The aspect ratio of the plate is $h=H / L=0.8$.

The first-order potential is shown in Figs. 9-13. The aspect ratios, the dimensions and the locations of the open areas are defined in the figure captions. The cases examined in the contour plots of Figs. 1013 are the same as those depicted in Figs. 5-8. As far as the first-order problem is concerned it is immediately apparent that the velocity potential does not comply with an explicit rule as regards its area as well as at the vertical edges of the plate. Figs 9 depicts sapsots of the potentin in both solid sections of the plate in 41 eqully spect points between $-1 \leq y \leq 1$. The firt exhibits vertically a type of pabolic var poin on the upper the potentia stats from a maximum at the flat bottom and progressively decays up to the first horizontal se from

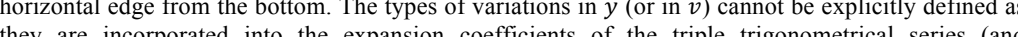
accordingly into the expansion coefficients of the integral equations).

The behaviour of the first-order velocity potential is better shown in the contour plots of Figs. 10-13. It is interesting to observe that the first-order component (which describes explicitly 3D effects) is nearly zero at the centreline of the plate in both solid sections of it. It is reminded that the leading order component has maxima on the centreline. Widening the gap causes a decrease of potential in both sections, while for larger aspect ratios the magnitudes of the potential are increased. The most interesting characteristic of the first-order component is that the maximum potential occurs far from the centre line and close to the vertical edges. The positions of maxima on either side of $y=0$, are the same for both solid sections and are detected at about $y= \pm 0.7$. Those findings clearly characterise the impact of the 3D effects. Indeed, those are important near the edges of the plate having some distance from them which secure the smooth reduction of the potential towards the vertical edges. For the higher aspect ratio case, the 3D effects due to the first-order potential appear to be more concentrated. In any event, the values of $\phi^{(1)}(0, y, z)$ are much smaller than $\phi^{(0)}(0, y, z)$ meaning that, as expected, the phenomenon is dominated by the leading order term.

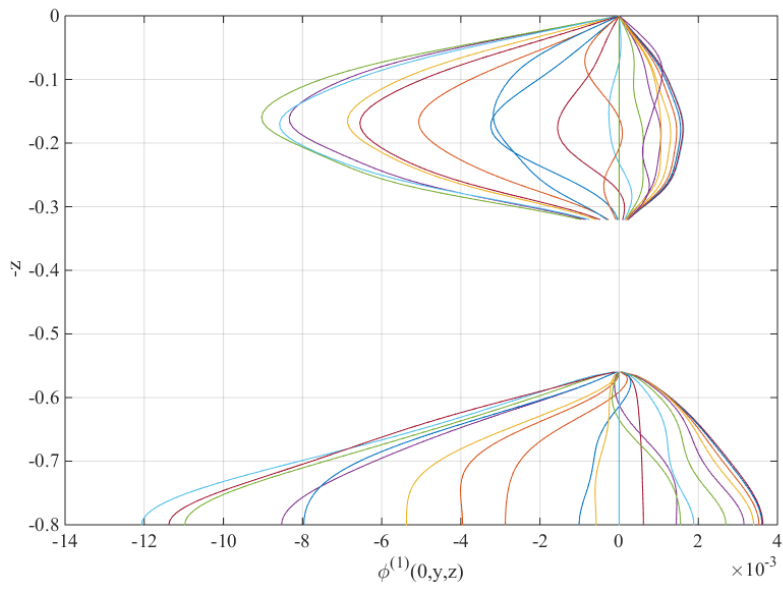

FIG. 9 The first-order potential $\phi^{(1)}(0, y, z)$ for lower edge $a / h=0.7$ and upper edge $b / h=0.4$. The aspect ratio of the plate is $h=H / L=0.8$.

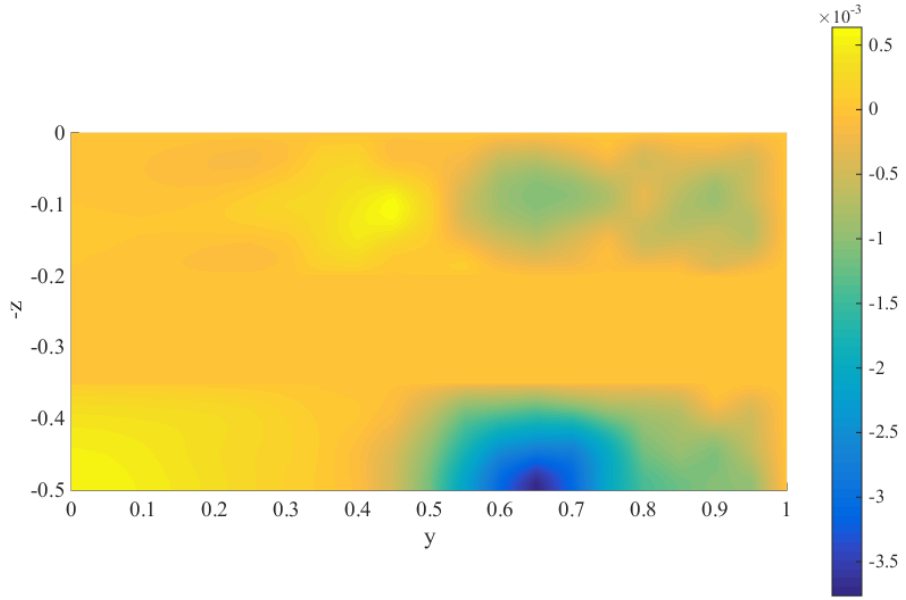

FIG. 10 The first-order potential $\phi^{(1)}(0, y, z)$ for lower edge $a / h=0.7$ and upper edge $b / h=0.4$. The aspect ratio of the plate is $h=H / L=0.5$. 


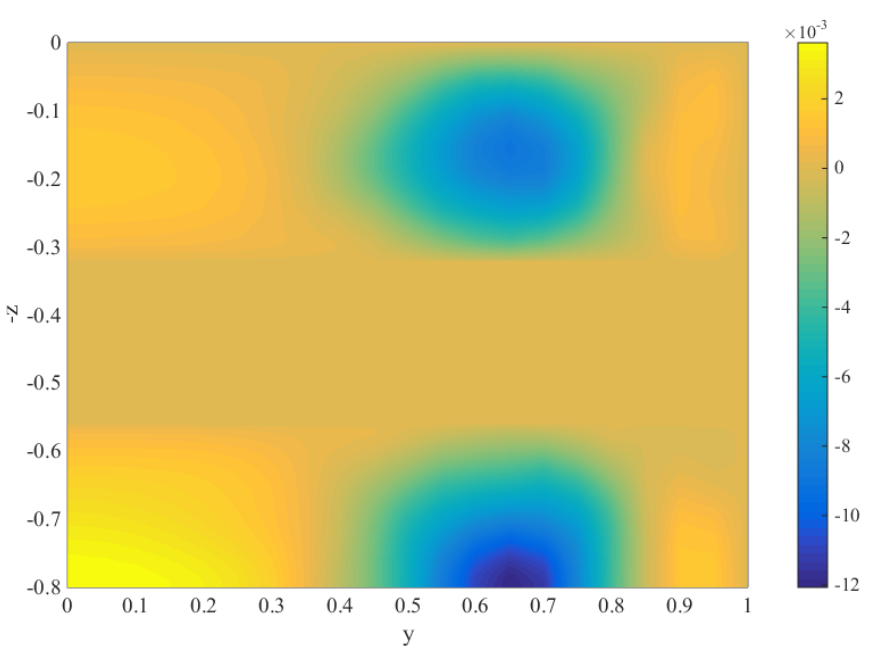

FIG. 11 The first-order potential $\phi^{(1)}(0, y, z)$ for lower edge $a / h=0.7$ and upper edge $b / h=0.4$ The aspect ratio of the plate is $h=H / L=0.8$.

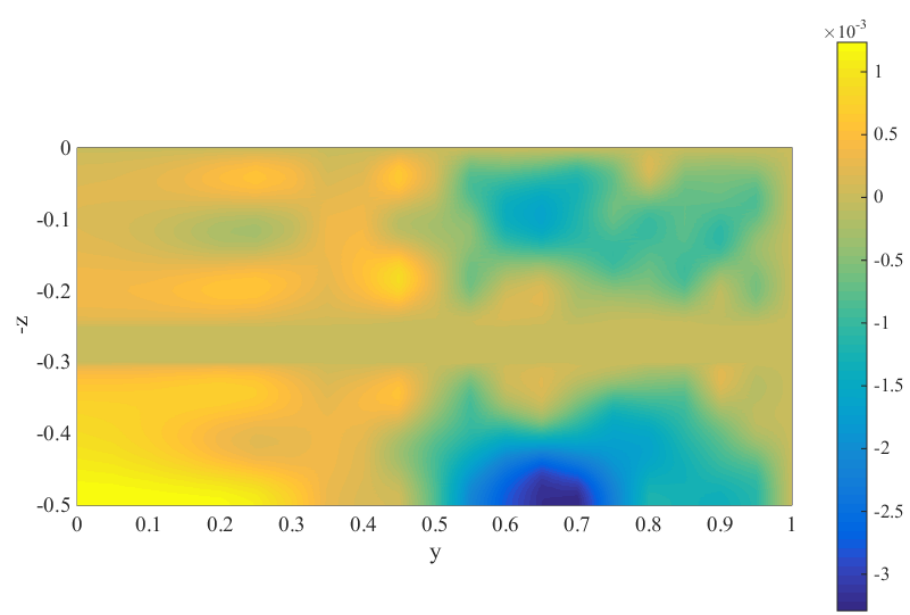

FIG. 12 The leading order potential $\phi^{(1)}(0, y, z)$ for lower edge $a / h=0.6$ and upper edge $b / h=0.5$. The aspect ratio of the plate is $h=H / L=0.5$.

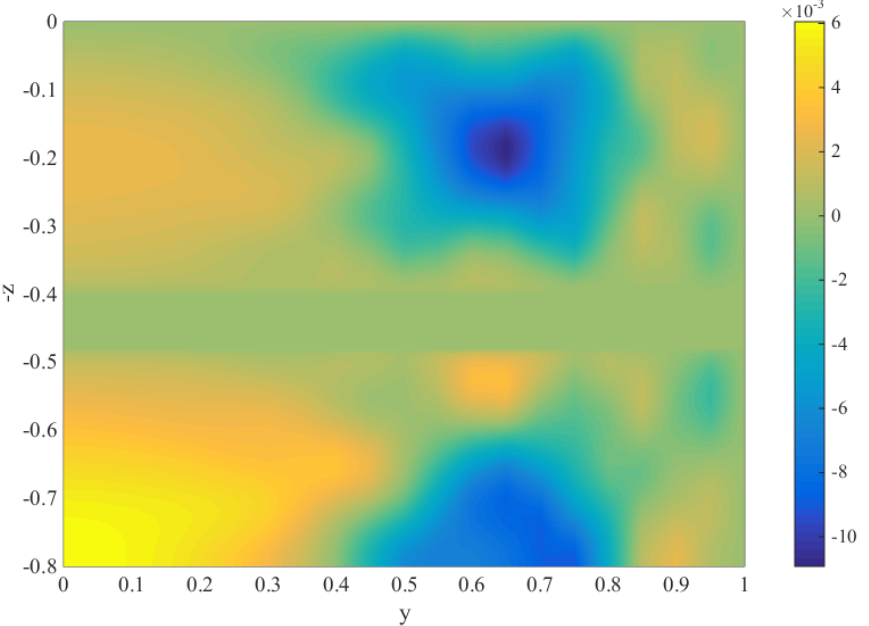

FIG. 13 The leading order potential $\phi^{(1)}(0, y, z)$ for lower edge $a / h=0.6$ and upper edge $b / h=0.5$. The aspect ratio of the plate is $h=H / L=0.8$.

\subsection{Pressure-impulse}

The phenomenon of hydrodynamic impact which involves a brief collision between a volume of liquid and a rigid body is better quantified by the so-called Pressure-Impulse, which is calculated by the short-lived pressures generated by the impact. According to Bagnold (1939), the pressure-impulse is approximately constant (among repetitions of waves) at a fixed point on the impacted wall, although the peak pressures change unpredictably from any one impact to the next. Also, in contrast to cordingly is a biter mone peak pressures (Richert, 1968).

Pressure-impulse has been mainly used to model flows induced instantaneously from rest, e.g. the impact of a rigid body striking the surface of still water (Batchelor, 1973; Cointe \& Armand 1987; impact of a rigid body striking the surface of still water (Batchelor, 1973, Cointe \& Armand, 1987; in a moving liquid domain that collides with a fixed structure, was introduced by Cooker and Peregrine (1995). Lamb (1932) defines the pressure-impulse as

$P(X, Y, Z)=\int_{0}^{\Delta t} p(X, Y, Z, t) d t$,

where the pressure $p(X, Y, Z, t)=-\rho \Phi_{t}(X, Y, Z, t), \rho$ is the water density and $\Delta t$ is the short duration of the impact. Accordingly, $P(X, Y, Z)=-\rho \Phi(X, Y, Z)$, where $\Phi(X, Y, Z)$ is the change in the velocity potential induced by the impact. The total impulse is thus obtained by integrating the impulsive pressures oven the total impacted area at $X=0$. Here, the total impulse, in normalized form, is given by 
$I(h)=-\int_{0}^{h} \int_{-1}^{1} \phi(0, y, z) d y d z$.

where $I(h)$ also depends on the parameters $a$ and $b$

The scale of the total impulse is $\rho V L^{3}$. The split in the potential suggests the split of the total impulse as well. Thus substituting (8.2) and (8.3) into (8.5) and performing the integrations we obtain the following expressions of the total impulse in the leading and the first-order:

$I^{(0)}(h)=-\frac{h^{2}}{2 \pi} \sum_{m=0}^{\infty} \sum_{n=1}^{\infty} \frac{(-1)^{m} A_{1}^{(2 m+1)}\left(q_{n}\right) S_{2 m+1}^{(n),(0)} M S_{2 m+1}^{\prime(3)}\left(0,-q_{n}\right)}{(n-1 / 2)^{2}}$,

$I^{(1)}(h)=-\frac{h^{4}}{2 \pi^{3}} \sum_{m=0}^{\infty} \sum_{n=1}^{\infty} \frac{(-1)^{m} A_{1}^{(2 m+1)}\left(q_{n}\right)\left[S_{2 m+1}^{(n),(1)} M s_{2 m+1}^{\prime(3)}\left(0,-q_{n}\right)-S_{2 m+1}^{(n),(0)} \Lambda_{2 m+1}^{(n)}\right]}{(n-1 / 2)^{4}}$.

Results for the total impulse for $h=0.5$ and 0.8 are shown in Figs. 14 and 15. Each figure contains both orders, while the total impulses are depicted as functions of $b / h$ for several values of $a / h$. The impulse at leading order is decreased from small to large openings. The decrease however is relatively smooth following a curved path while the total impulse at leading order tends to an asymptotic constant as the height of the upper section obtains very small values.

The first-order component of the total impulse is more interesting. It starts from zero for wide openings while for the small aspect ratio case exhibits a maximum which however does not coincide Wh the case of narrow opening. For the large aspect ratio case, that maximum disappears. specific case the total impulse becomes negative. Finally, it can be safely concluded that the leading order total impulse is the dominot component as the first-order counterpart is literarily negligible

\section{Conclusions}

This study dealt with the 3D hydrodynamic impact problem on a vertical plate with a rectangular opening due to the impact of a steep wave. The solution method employed linear potential theory
while the hydrodynamic problem was formulated as a boundary value problem of mixed type. The model equations defined two MBVPs the two directions of the plate. The first MBVP was tackled assimilating the plate as a degenerate elliptical cylinder with negligible semi-minor axis. That allows the explicit satisfaction of the Dirichlet conditions of the change in the velocity potential (due to the brief impact) beyond the edges of the plate in the horizontal direction. Accordingly, the Neumann and the Dirichlet conditions on the solid parts of the plate and its opening, respectively, defined a new MBVP in the vertical direction involving triple trigonometrical series.

To achieve a solution for the latter MBVP, initially a perturbations technique was employed that defined components at various orders. The modified triple trigonometrical series MBVP was treated using a novel methodology that determined expressions in the form of triple integral equations. The sought solution was achieved by reducing the triple integral equations into dual trigonometrical series.

We presented results for the velocity potential that concerned both the leading order and the firstorder components. As expected, the former was found to be much larger than the latter. The dominan leading order velocity potentials exhbited smooth variations both horizontally and vertically. It was also found that the existence of an opening along the plate's height, even very small, results in drastic reduction of the change in the velocity potential due to the hydrodynamic impact. The increase in the plate's aspect ratio leads to the increase in the magnitude of the potential. The contour lines of the velocity potential were found to be elliptical in the upper solid section of the plate while their geometry on the lower section resembles half-ellipses. The maxima of the leading order component were always occurred along the plate's centreline. In contrast, the first-order potential that is dominated by the 3D effects was zero on the centreline and its maxima were concentrated close to the edges of the plate in the form of concentric circles on the upper solid section and semi-circles in the lower section.

Finally, pressure impulse theory was employed to evaluate the pressure impulse due to the change in the potential at the instant of impact. In connection with pressure impulse, it was found that the leading order pressure impulse is reduced by widening the opening, following however a curved path. In contrast, the significantly smaller first-order pressure impulse exhibits a complicated variation, while for very small openings becomes negative.

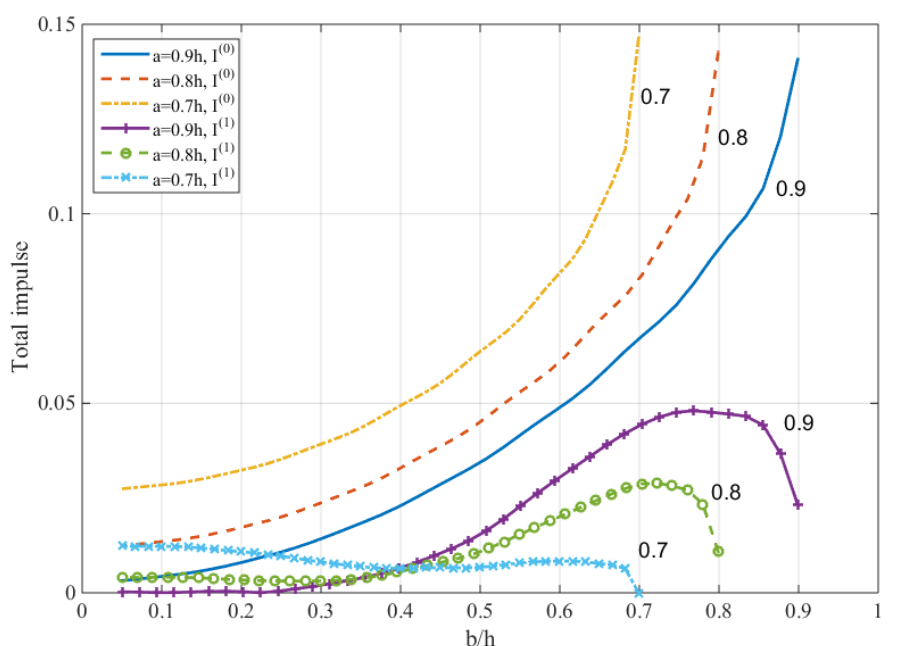

FIG. 14 Total impulses $I^{(0)}$ and $I^{(1)}$ as functions of $b / h$ for several values of $a / h$. The aspect ratio of the plate is $h=H / L=0.5$. For display purposes the total impulse at first-order has been multiplied by 50 . 


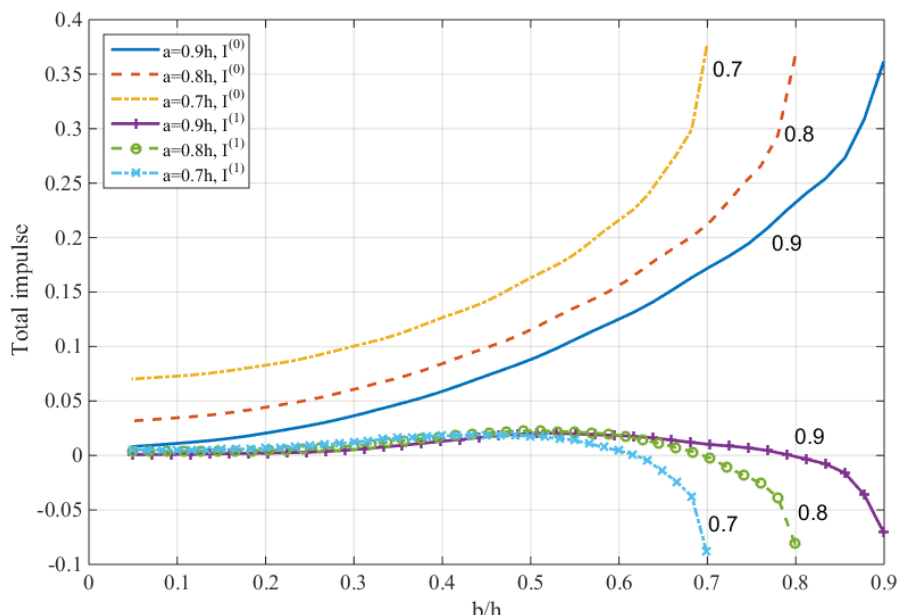

FIG. 15 Total impulses $I^{(0)}$ and $I^{(1)}$ as functions of $b / h$ for several values of $a / h$. The aspect ratio of the plate is $h=H / L=0.8$. For display purposes the total impulse at first-order has been multiplied
by 20 .

Acknowledgements. The authors are grateful to the EU Marie Curie Intra European Fellowship project SAFEMILLS "Increasing Safety of Offshore Wind Turbines Operation: Study of the violen wave loads" under grant 622617.

\section{REFERENCES}

AbramowitZ, M. \& Stegun, I.A. (1970) Handbook of Mathematical Functions. New York: Dover Publications Inc.

BATCHELOR, G.K. (1973) An Introduction to Fluid Dynamics. Cambridge: Cambridge University

Press.
BAGNOLD, R.A. (1939) Interim report on wave pressure research. Journal of Institution of Civil Engineers, 12, 201-226.

Bullock, G.N., Obharai, C., Peregrine, D.H. \& Bredmose, H. (2007) Violent breaking wave impacts. Part 1: Results from large-scale regular wave tests on vertical and sloping walls. Coastal Engineering, 54, 602-617.

BREDMOSE, H., PEREGRINE, D.H. \& BULLOCK, G.N. (2009) Violent breaking wave impacts. Part 2: modelling the effect of air. Journal of Fluid Mechanics, 641, 389-430.

BREDMOSE, H., BULLOCK, G.N. \& HOGG, A.J. (2015) Violent breaking wave impacts. Part 3. Effects of scale and aeration. Journal of Fluid Mechanics, 765, 82-113.

CARRATELLI, E.P., VICCIONE \& G., BOVOLIN, V. (2016) Free surface flow impact on a vertical wall: a numerical assessment. Theoretical and Computational Fluid Dynames, DOI 10.1007/s00162-

ASME: Journal of Offshore Mechanics and Arctic Engineering, 109, 237-243.

COINTE, R. (1989) Two-dimensional water-solid impact. Transactions ASME. Journal of Offshore Mechanics and Arctic Engineering, 111, 1090-114.
CoOKer, M.J. \& Peregrine, D.H. (1995) Pressure-impulse theory for liquid impact problems. Journal of Fluid Mechanics, 297, 193-214.

COOKER, M.J. (2013) A theory for the impact of a wave breaking onto a permeable barrier with jet generation. Journal of Engineering Mathematics, 79, 1-12.

CuMmins, S.J., SILVESTER, T.B. \& CLEARY, P.W. (2012) Three-dimensional wave impact on a rigid structure using smoothed particle hydrodynamics. International Journal for Numerical Methods in Fluids, 68, 1471-1496.

CUOMO, G., AlLSOP, W., BRUCE, T. \& PEARSON, J. (2010) Breaking wave loads at vertical seawalls and breakwaters. Coastal Engineering, 57, 424-439.

DufFY, D.G. (2008) Mixed Boundary Value Problems. Boca Raton, FL: Chapman \& Hall/CRC.

GÓMEZ-GESTEIRA, M \& DALRYMPLE, R.A. (2004) Using a three-dimensional smoothed particle hydrodynamics method for wave impact on a tall structure. Journal of Waterway, Port, Coastal and Ocean Engineering, 130, 63-69.

FABRIKANT V.I. (1991) Mixed Boundary Value Problems of Potential Theory and Their Applications in Engineering. Dordrecht: Kluer Academic Publishers.

GRADSHTEYN, I.S. \& RYZHIK, I.M. (2007) Table of Integrals, Series and Products (7th edition). London: Elsevier Academic Press.

HOWISON, S.D., OCKENDON, J.R. \& WILSON, S.K. (1991) A note on incompressible water entry problems at small dead-rise angles. Journal of Fluid Mechanics, 222, 215-230.

Kerr, G., Melrose, G. \& TWeEd, J. (1994) Some triple sine series. Applied Mathematics Letters, 7, 33-36.

KING, A.C. \& NEEDHAM, D.J. (1994) Initial development of a jet caused by fluid, body and freesurface interaction. Part 1. A uniformly accelerating plate. Journal of Fluid Mechanics, 268

KleEFsMAN, K.M.T., FekKen, G., Veldman, A.E.P., IWANowski, B. \& Buchner, B. (2005) A Volume-of-Fluid based simulation method for wave impact problems. Journal of Computational Physics, 206, 363-393.

KorobKin, A.A \& SCOLAN, Y.-M. (2006) Three-dimensional theory of water impact. Part 2. Linearized Wagner problem. Journal of Fluid Mechanics, 549, 343-373.

KOROBKIN, A.A \& MALENICA, Š. (2007) Steep wave impact onto elastic wall Proc $22^{\text {nd }}$ International Workshop on Water Waves and Floating Bodies, Plitvice, Croatia, 129-132.

KOROBKIN, A.A. (2008) Non-classical boundary conditions in water-impact problems. IUTAM Symposium (ed. E. Kreuzer), 167-178.

MAGNUS, W. \& OBERHETTINGER, F. (1949) Formulas and Theorems for the Special Functions of Mathematical Physics: New York: Chelsea.

MOON, P. \& SPENCER, D.E. (1971) Field Theory Handbook. Berlin: Springer-Verlag.

NOAR, N.A.Z.M \& GREENHOW, M. (2015) Wave impacts on structures with rectangular geometries: Part 1. Seawalls. Applied Ocean Research, 53, 132-141

PEREGRINE, D.H. (2003) Water-wave impact on walls. Annual Review of Fluid Mechanics, 35, 23-43. RAFIEE, A., DUTYKH, D. \& DIAS, F. (2015) Numerical simulation of wave impact on a rigid wall using a two-phase compressible SPH method. Procedia IUTAM, 18, 123-137.

RICHERT, G. (1968) Experimental investigation of shock pressures against breakwaters. Proc. 11th Conference on Coastal Engineering, ASCE, 945-973.

SCOLAN, Y.-M. \& KOROBKIN, A.A. (2001) Three-dimensional theory of water impact. Part 1. Inverse Wagner problem. Journal of Fluid Mechanics, 440, 293-326.

SCOLAN, Y.-M. (2014) Hydrodynamic impact of an elliptic paraboloid on cylindrical waves. Journal of Fluids and Structures, 48, 470-486.

SHEPHERD, W.M. (1938) On trigonometrical series with mixed conditions. Proc. London

Mathematical Society, 43, 366-375.
SNEDDON, I.N. (1966) Mixed Boundary Value Problems in Potential Theory. Amsterdam: North Holland Publishing Company.

SRIVASTAV R.P. (1963) Dual series relations. IIL. Dual relations involving trigonometric series. Proc. Royal Society Edinburgh, 66, 173-184. 
TITCHMARSH, E.C. (1948) Introduction to the Theory of Fourier Integrals (2nd edition). Oxford: Clarendon Press.

Pual trigonometrical series, Glasgow Mathematical Journal, 4, 49-57.

A A Tual trigonometrical series. Glasgow Mathematical Journal,

RANTER, C.J. (1960b) Some triple integral equations. Glasgow Mathematical Journal, 4, 200-203.

TRANTER, C.J. (1964) An improved method for dual trigonometrical series. Glasgow Mathematical Journal, 6, 136-140.

TRANTER, C.J. (1969) Some triple trigonometrical series. Glasgow Mathematical Journal, 10, $121-$ 125 .

Wagner, H. (1932) Über Stross- und Gleitvorgänge an der Oberfläche von Flüssigkeiten. Z. Angew. Math. Mech., 12, 193-215. (English transl: Phenomena associated with impacts and sliding on liquid surfaces. NACA Translation 1366)

Watson, G.N. (1944) A Treatise on the Theory of Bessel Functions. Cambridge: Cambridge University Press.

WILLIAMS, W.E. (1963) Note on the reduction of dual and triple series equations to dual and triple integral equations. Proc. Cambridge Philosophical Society, 59, 731-734.

WoOD, D., Peregrine, D.H. \& BRUCE, T. (2000) Wave impact on a wall using pressure-impulse theory: I: Trapped air. Journal of Waterway, Port, Coastal and Ocean Engineering, 126, 182190.

WOOD, D. \& PEREGRINE, D.H. (2000) Wave impact on a wall using pressure-impulse theory: II: Porous berm. Journal of Waterway, Port, Coastal and Ocean Engineering, 126, 191-195.

YANG, C., LIN, B., JIANG, C. \& LIU, Y. (2010) Predicting near-field dam-break flow and impact force 